

\section{DISCLAIMER}

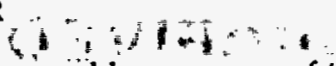

This document was prepared as an account of work sponsored by an agency of the United States Government. Neither the United States Government nor the University of California nor any of their employees, makes any warranty, express or implied, or assume any legal liability or responsibility for the accuracy, completeness, or usefulness of any information, apparatus, product, or process disclosed, or represents that its use would not infringe privately owned rights. Reference herein to any specific commercial product, process, or service by trade name, trademark, manufacturer, or otherwise, does not necessarily constitute or imply its endorsement, recommendation, or favoring by the United States Government or the University of California. The views and opinions of authors expressed herein do not necessarily state or reflect those of the United States Government or the University of California, and shall not be used for advertising or product endorsement purposes.

This report has been reproduced directly from the best available copy.

Available to DOE and DOE contractors from the Office of Scientific and Technical Information

P.O. Box 62, Oak Ridge, TN 37831

Prices available from (615) 576-8401, FTS 626-8401

Available to the public from the

National Technical Information Service

U.S. Department of Commerce

5285 Port Royal Rd.

Springfield, VA 22161 


\section{DISCLAIMER}

Portions of this document may be illegible in electronic image products. Images are produced from the best available original document. 


\title{
Optimization of the Mini-Flo Flow Cytometer
}

\author{
by \\ Mukund C. Venkatesh \\ B.S. Mechanical Engineering \\ Massachusetts Institute of Technology, 1995
}

SUBMITTED TO THE DEPARTMENT OF MECHANICAL ENGINEERING IN

PARTIAL FULFILLMENT OF THE REQUIREMENTS FOR THE DEGREE OF

MASTER OF SCIENCE IN MECHANICAL ENGINEERING

AT THE

MASSACHUSETTS INSTITUTE OF TECHNOLOGY

JUNE 1996 


\title{
Optimization of the Mini-Flo Flow Cytometer
}

\author{
by \\ Mukund C. Venkatesh \\ Submitted to the Department of Mechanical Engineering \\ on May 25, 1996 in Partial Fulfillment of the \\ Requirements for the Degree of Master of Science in \\ Mechanical Engineering
}

\begin{abstract}
:
A new method of collecting light scattering from a liquid flow cytometer has been proposed [Mariella, 1995]; this apparatus is named the Mini-Flo flow cytometer. The Mini-Flo uses a high numerical aperture collection immersed in the flow stream. The collector consists of a conically tipped fiber optic pipe and terminating optical detector. This study was performed to improve the signal/noise ratio and optimize the Mini-Flo's performance for HIV blood detection applications [Vyas, 1994].

Experiments were performed to gauge the effects of Raman scattering, lens/filter fluorescence, and fiber optic fluorescence on the Mini-Flo's performance and signal/noise ratio. Results indicated that the fiber optic was a major source of fluorescence noise and reducing its length from $13^{\prime \prime}$ to $3^{\prime \prime}$ increased the signal/noise ratio from 8 to 75 . Therefore, one of the key issues in optimizing the Mini-Flo's performance is a redesign of the holding structure such that the fiber optic length is minimized. Further improvements of the Mini-Flo's performace can be acheived by studying the polish of the fiber optic, the flow over the fiber optic's conical tip, and the optimal particle rates.
\end{abstract}

Thesis Supervisor: Dr. Raymond Mariella Jr.

Title: Company Supervisor

Thesis Supervisor: Shaoul Ezekiel

Title: Professor of Aero Astro \& Eecs 


\section{Acknowledgments}

I would like to first thank Ray Mariella, my supervisor at the Lawrence Livermore National Laboratories, for giving me the opportunity to work on such an interesting project. Without his guidance and support, I would have not been able to have such a great experience. Don Masquelier, the primary mechanical engineer working on this project at the Lab, took me under his wing and explained all the details of the Mini-Flo. He helped me with the entire experimentation process and for that I am grateful. I would also like to thank Gerald Eveleth and Alex Copeland for their constant help and advice during my term at the Lab.

Professor Shaoul Ezekiel was a mentor throughout the entire project. Even though he was not directly supervising the project, Prof. Ezekiel's input was invaluable. It is often with reservations that professors agree to sponsor a company thesis, as the majority of the work is done away from campus. I. thank him for taking the time to supervise my thesis on the MIT side.

I could not leave MIT alive without thanking some of my good friends who helped me make it through my five years at the Institute including Dany Saliba Cheij, Dexter Mootoo, Kuniaki Takahashi, Hans Jacob Feder, and Mehran Islam.

In addition, I am deeply indebted to my parents, Mandyam and Meera Venkatesh, and my brother Sridhar Venkatesh. It is impossible to put in words the love, understanding, and support they have given me over the years.

Finally, I would like to extend gratitude and apologies to all the people I have bugged, annoyed, or pestered in the completion of this thesis. 


\section{Table of Contents}

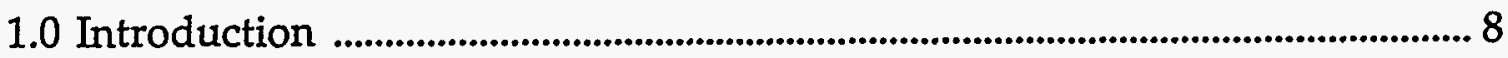

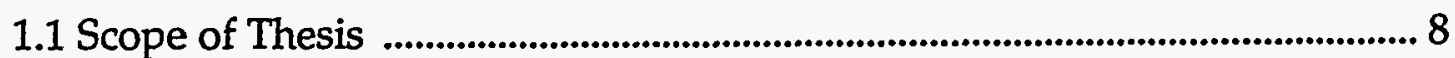

1.2 What is Flow Cytometry ..................................................................................... 9

1.3 How Does Flow Cytometry Work ....................................................................... 11

1.3.1 Light Scattering - Elastic and Inelastic Light ........................................... 11

1.3.1.1 Elastic Scattered Light ........................................................................... 12

1.3.1.2 Inelastic Scattered Light .......................................................................... 15

1.3.2 Excitation Sources ............................................................................................. 18

1.3.3 Light Collection Systems and Detection .................................................. 19

1.3.4 Flow System ............................................................................................................ 21

1.3.5 Electronics and Signal Processing ............................................................. 22

1.4 The Mini-Flo Flow Cytometer ...................................................................... 24

1.4.1 Mini-Flo vs. Conventional Flow Stream in Air Flow Cytometers 26

1.4.1.1 Advantages ................................................................................................ 26

1.4.1.2 Disadvantages ........................................................................................... 29

1.4.2 Mini-Flo and Possible Applications ........................................................ 30

2.0 The Mini-Flo: Detailed Design Discussion .............................................................. 31

3.0 Signal/Noise Ratio Issues ........................................................................................ 33

3.1 PMT Performance ...................................................................................................... 33

3.2 Lenses, Filters, and Fiber Optics ...................................................................... 34

3.2.1 Optical Leakage ....................................................................................................... 35

3.2.2 Optic Auto-Fluorescence .............................................................................. 36

3.3. Raman Scattering ......................................................................................... 37

3.4 Miscellaneous Noise Sources ................................................................................. 37

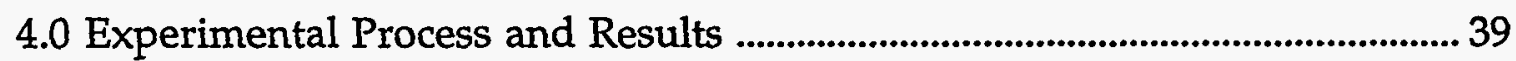

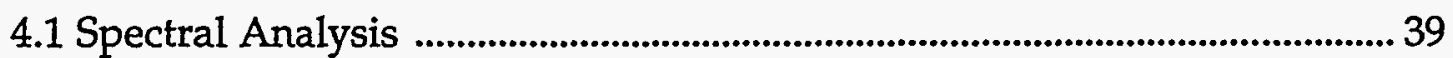

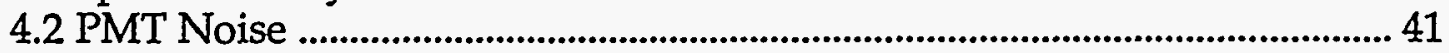

4.3 Laser Purity and Optical Leakage ...................................................................... 43

4.4 Optical and Fiber Optic Auto-Fluorescence .................................................... 46

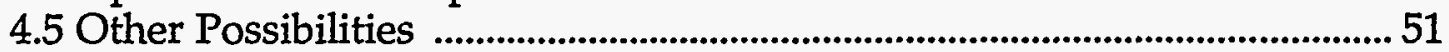

4.5.1 Random Particles in Sheath ............................................................. 51

4.5.2 Ambient Light ................................................................................................. 52

4.5.3 Electronic Noise ......................................................................................... 52 
5.0 Discussion and Other Issues............................................................................... 54

5.1 The New Mini-Flo (A Design Discussion) ..................................................... 55

5.2 Issues for Further Study .................................................................................... 58

5.2.1 Fiber Optic End Polishing ….......................................................................... 58

5.2.2 Flow Over Conical Tip .............................................................................. 60

5.2.3 Particle Rates ......................................................................................................... 61

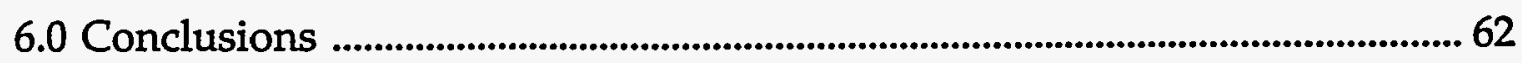

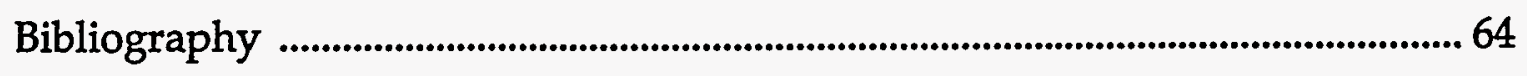




\section{List of Figures}

Figure 1.1 - Conventional flow cytometer ............................................................... 10

Figure 1.2 - Geometry for particle scattered light ................................................. 13

Figure 1.3 - Mie scattered light patterns ................................................................... 14

Figure 1.4 - Scattered light interference patterns ........................................................ 15

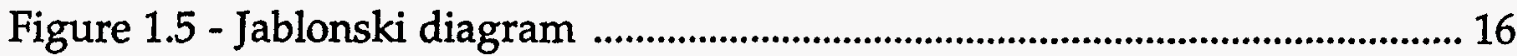

Figure 1.6 - Fluorescence spectrum of fluorescein .................................................. 17

Figure 1.7 - Observation point ............................................................................. 19

Figure 1.8 - Flow nozzle ............................................................................................ 21

Figure 1.9 - Mini-Flo waveguide system ................................................................. 25

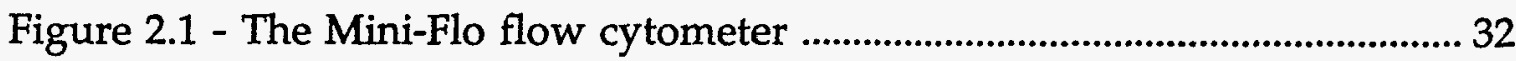

Figure 2.2 - Optics package schematic ................................................................... 32

Figure 3.1 - Dark current signals vs. Fluorescence signals .................................... 34

Figure 3.2 - Percent reflectivity characteristic of dichroic .................................... 36

Figure 4.1 - Wavelength scans for Polyscience beads ............................................ 40

Figure 4.2 - Wavelength scans for Raman scattering of water ............................ 41

Figure 4.3 - PMT dark current photo ................................................................. 42

Figure 4.4 - PMT with laser on ...................................................................... 42

Figure 4.5 - PMT with water and laser on .......................................................... 43

Figure 4.6 - Oscilloscope signals of Polyscience fluorescent .................................. 45 and non-fluorescent beads with Chroma $530 / 50 \mathrm{~nm}$ bandpass filter

Figure 4.7 - Oscilloscope signals of Polyscience fluorescent 45 and non-fluorescent beads with Chroma $530 / 50 \mathrm{~nm}$ bandpass and $510 \mathrm{~nm}$ longpass filter 
Figure 4.8 - Oscilloscope signals of Polyscience fluorescent 46 and non-fluorescent beads with Chroma $530 / 50 \mathrm{~nm}$ bandpass and two $510 \mathrm{~nm}$ longpass filters

Figure 4.9 - Illustration of filter auto-fluorescence 48

Figure 4.10 - Oscilloscope signals of Polyscience non-fluorescent beads 48 with a 1 " distance

Figure 4.11 - Oscilloscope signals of Polyscience non-fluorescent beads 48 with a $3^{\prime \prime}$ distance

Figure 4.12 - Oscilloscope signals of FCS fluorescent and Polyscience 49 non-fluorescent beads with $13^{\prime \prime}$ fiber optic

Figure 4.13 - Oscilloscope signals of FCS fluorescent and Polyscience 50 non-fluorescent beads with $3^{\prime \prime}$ fiber optic

Figure 5.1 - Modified optics package schematic 56

Figure 5.2 - Modified electronics schematic 57

Figure 5.3 - Ideal fiber optic conical polish 59

Figure 5.4 - Actual fiber optic conical polish 59 


\subsection{Introduction}

\subsection{Scope of Thesis}

The focus of this thesis originates from an application suggested by Dr. Vyas, a hematologist blood bank expert at the University of California, San Francisco. An immunoreactive bead-based assay which probes for four different HIV nucleic-acid sequences has been invented. The biological preparation of the blood sample is quite complex, and the final sample for Mini-Flo processing is a solution of four different size Dynal magnetic beads ranging in diameter from $1 \mu \mathrm{m}$ to $10 \mu \mathrm{m}$. Each bead size is dedicated to a specific HIV sequence, and if it is present in the sampled blood, the bead accumulates fluorescenated DNA from the PCR process and fluoresces. Thus, an HIV positive blood sample will be read by Mini-Flo as four different scatter sizes with positive fluorescence signals on each one. The main advantages of this detection scheme are reliability and speed. Presently, HIV detection is a timely process occasionally resulting in false positive or negative results. A bead-based assay such as the one described above is easy to prepare and, because it is quadrupolly redundant, very reliable. This application requires an inexpensive flow cytometer with a fluorescence sensitivity level greater than or equal to the commercially available FacScan. In particular, the magnitude of a fluorescence signal must be 10 to 100 times greater than a nonfluorescence signal with zero overlap between the weaker fluorescence signal 
and the stronger non-fluorescence signal. The reason for these strict requirements is to ensure accuracy when diagnosing HIV. Presently, the Mini-Flo provides a gain of about 8 between fluorescent and non-fluorescent beads. Therefore, an in depth study of background, noise sources, and light collection needs to be performed to increase the signal/noise ratio, and deem it useful for HIV blood detection as well as make' a significant positive impact on research in immunohematology.

The goal of this thesis was to perform an in depth study of all sources of background and noise in the Mini-Flo and suggest what can be done to improve the signal/noise ratio. The thesis covers the background, results, and conclusions of this study. In addition, a new design is proposed for the MiniFlo, and suggestions are given for further studies to improve the Mini-Flo's performance.

\subsection{What is Flow Cytometry?}

Cytometry refers to the measurement of physical and/or chemical characteristics of cells, or, by extension, of other biological particles. Flow cytometry is a process in which individual cells, or other biological particles, are made to pass, in single file in a fluid stream, by a sensor or sensors which measure physical or chemical characteristics of the cells or particles [Shapiro, 1995]. 
A conventional flow cytometer schematic is illustrated in Figure 1.1 [Shapiro, 1995]. Particles are channeled single file, and a laser beam is focused onto the flowing particles, and light scatter detectors are placed both in front of the laser (forward scattered light) and perpendicular to the laser line (right angle scattered light - RAS). These light scatter detectors provide information corresponding to the size, shape, and chemical composition (by way of cytochemical fluorescent probes) of the particle.

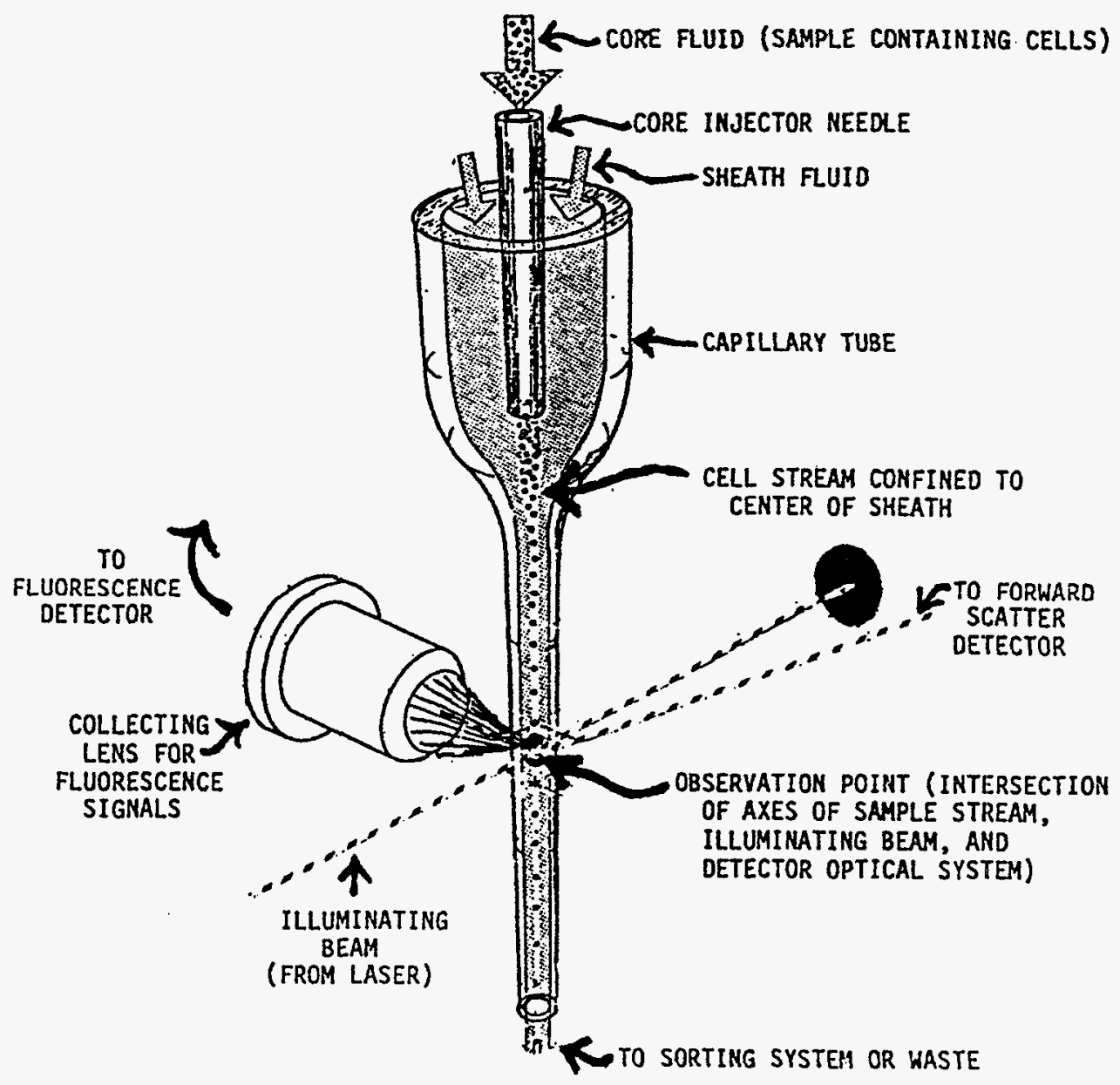

Figure 1.1 - Conventional Flow Cytometer 


\subsection{How Does Flow Cytometry Work?}

Flow cytometry of biological specimens became an experimentally viable process in the 1940 's. As with all relatively new fields, the rapid progress of flow cytometry can be attributed mainly to empirical successes. Experts, however, are now beginning to unravel the messages of light scattering signals at the laser-particle intersection, in the hopes of further advancing flow cytometry. The following section will briefly summarize what is currently known about flow cytometry on a theoretical level.

\subsubsection{Light Scattering - Elastic and Inelastic Light}

Despite the vast amount of information contained in light-scattering signals from particles generated by flow cytometers, meaningful deconvolution of that information continues to be a problem. As the title suggests, scattered light can be broken into two major categories: elastic and inelastic scattered light. In the case of elastic scattered light, the light signals have the same wavelength as the incident light; inelastic scattered light deals with light signals which have a wavelength shift from the incident light. 


\subsubsection{Elastic Scattered Light}

In order to understand the phenomenon of elastically scattered light, one must consider the effect of an electromagnetic light wave upon a spherical parcel of matter. If this sphere is small compared to the wavelength of the light, the oscillating electrical field associated with the radiation will, at any particular time, be uniform over the extent of the sphere. The electrons in this matter are driven by the incident electrical field and will therefore oscillate at the same frequency and reradiate electromagnetic waves in all directions. It is this redirection of the incident radiation that constitutes the elastic scattering process, called Rayleigh scattering. Consider that a small spherical isotropic particle located at the origin of a rectangular coordinate system is illuminated by a plane polarized electromagnetic wave of wavelength $\lambda$, which propagates in the $z$ direction (see Figure 1.2). An observer $\mathrm{O}$ at polar coordinates $(r, \theta)$ in the $x y$ plane will observe scattered radiation at the same wavelength $\lambda$ whose intensity will be

$$
\left(\begin{array}{l}
I_{1} \\
I_{2}
\end{array}\right)=\frac{128 \pi^{5}}{3 r^{2} \lambda^{4}} \alpha^{2}\left(\begin{array}{c}
1 \\
\cos ^{2} \theta
\end{array}\right)\left(\begin{array}{l}
I_{1}^{0} \\
I_{2}^{0}
\end{array}\right)
$$

where $\alpha$ is termed the electric polarizability. For a molecule, $\alpha$ is an intrinsic property, given by

$$
\alpha=a^{3}\left(m^{2}-1\right) /\left(m^{2}+2\right)
$$


where $a$ is the radius of the particle and $m$ is its refractive index. In each case, $\alpha$ may depend upon the wavelength. These expressions must be modified somewhat when the particle is anisotropic or nonspherical. $I_{1}^{0}$ and $I_{1}$ represent the intensity of the incident and scattered light whose electric vector is perpendicular to the scattering (yz) plane; $I_{2}^{0}$ and $I_{2}$ represent parallel polarization [Kerker, 1983].

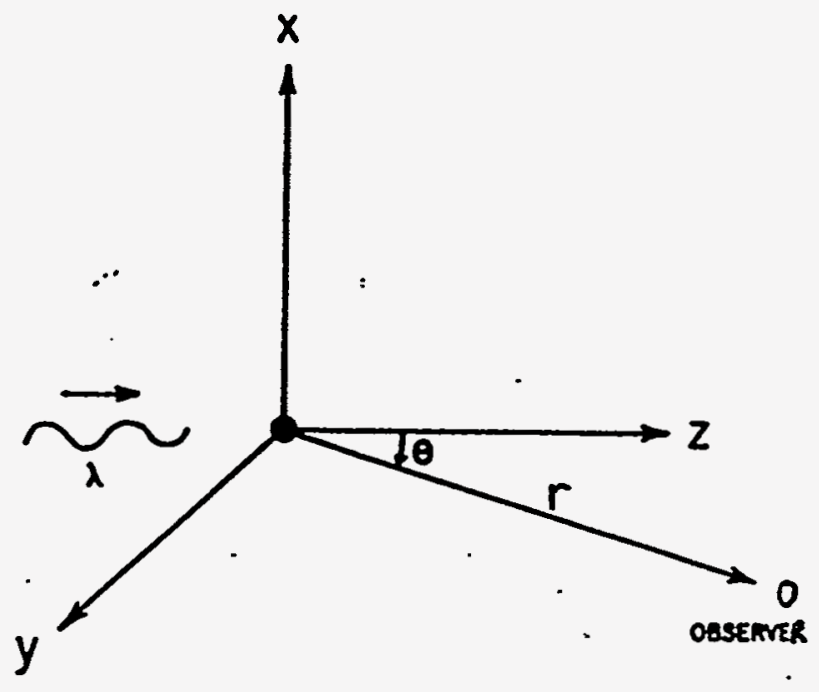

Figure 1.2 - Geometry for particle scattered light

If the particle diameter is greater than or equal to the incident wavelength, the scattered radiation can no longer be described by Rayleigh scattering. As the particles become larger, interference patterns become an issue. This is Mie scattering, named after Gustav Mie, who worked out the relevant theory using the laws of reflection, refraction, and diffraction. Mie scattering is a complex phenomenon influenced by numerous factors, a discussion of which extends beyond the scope of this thesis. According to Mie 
scattering, Figure 1.3 illustrates the possible paths of a light ray incident on a spherical particle. With these possible paths of light that can be traced from an incident bundle of rays, scattering interference patterns develop as illustrated in Figure 1.4. These interference patterns are unique to the shape and size of the particle, and the incident light wavelength. One might expect that this would provide a magnificent fingerprint for precise identification but this is not the case. For spheres, there are so many closely spaced maxima and minima that practical detectors in any instrument integrate the signals over a range of scattering angles, thereby washing out most of the structure. In addition, whenever such a bundle of rays is incident upon a curved surface, the intensity will be altered by the spreading of the rays upon reflection and refraction and this entails approximations that break down either for particles that are insufficiently large or for particular bundles of rays such as those that give rise to the rainbow [Kerker, 1983].

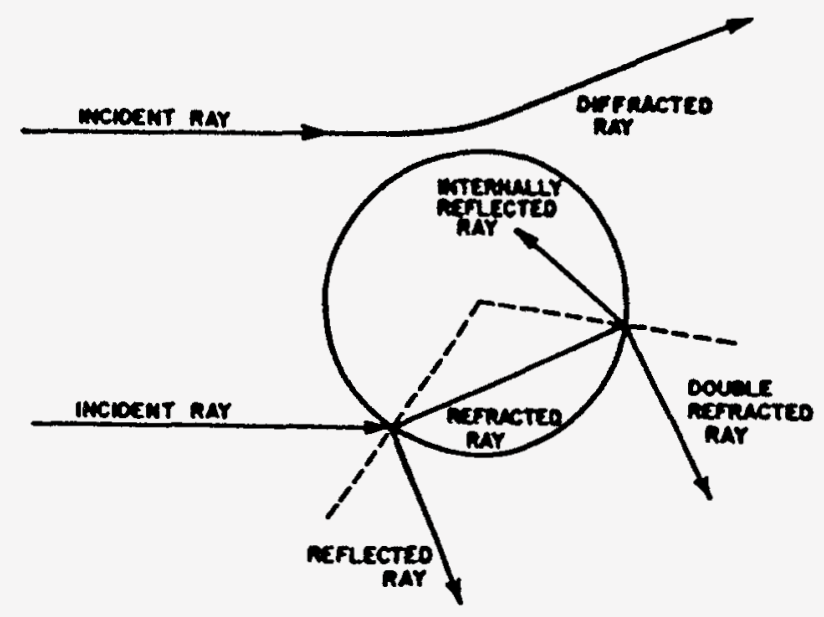

Figure 1.3 - Mie scattered light patterns 


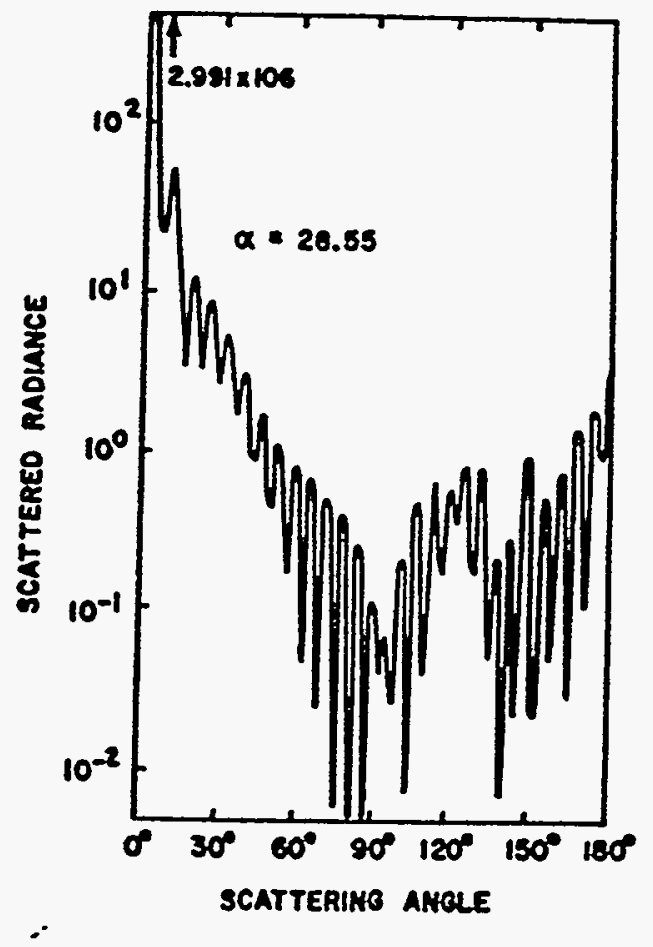

Figure 1.4 - Scattered light interference patters

In the case of flow cytometry, the primary angles of concern are 90 and 0 degrees - right angle and forward scattering, respectively. The forward scattered light serves primarily as a trigger signal. When the amplitude of the trigger signal exceeds a threshold level set by the user, a measurement in the right angle direction is initiated, so that deductions may be made about the size and shape of the particle.

\subsubsection{Inelastic Scattered Light}

As mentioned before, inelastic scattered light deals with the large category of scattered light that experiences a wavelength shift from the 
incident light. Absorption of UV or visible light leaves particles in excited electronic states; Figure 1.5, the Jablonski diagram, illustrates the number of mechanisms by which these particles can get out of excited states, and transition back to the electronic ground state [Lansing, 1986]. Flow cytometry is primarily concerned with the fluorescence transition and its detection.

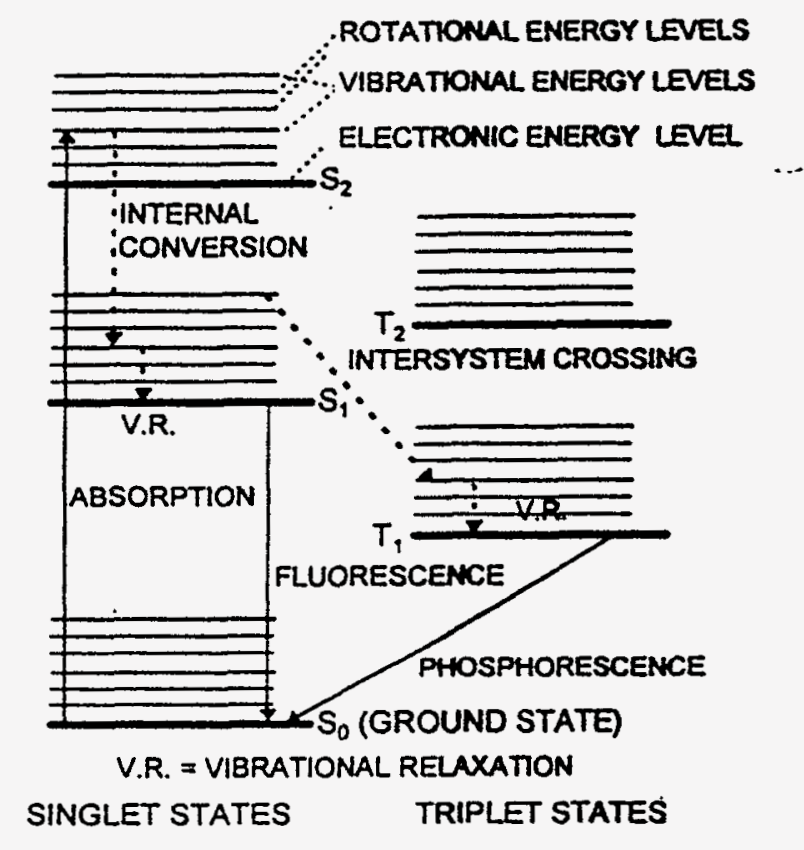

Figure 1.5- Jablonski diagram

Fluorescence emissions in general occur at longer wavelengths than the absorption which precedes it. This wavelength difference between excitation and emission is known as the Stokes shift. Figure 1.6 illustrates the Stokes shift of fluorescein, a common fluorescent marker used in flow cytometry. Fluorescence detection in flow cytometry opens the user to an entirely new dimension of information collection besides particle size and 
morphology. With fluorescence detection, the flow cytometrist can probe for various chemical and biological substances.

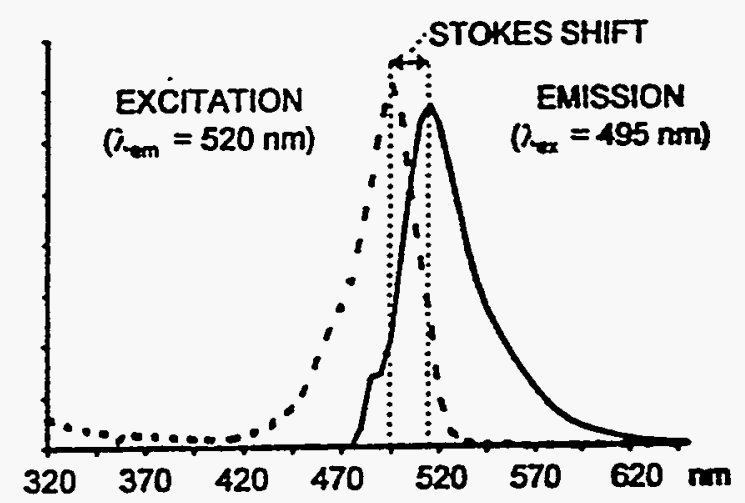

Figure 1.6 - Fluorescence spectrum of fluorescein

In addition to fluorescence, flow cytometry is also concerned with Raman scattering, another form of inelastic scattering. Raman scattering describes the phenomenon where molecules undergo a vibrational transition at precisely the time scattering occurs. This results in the emission of a photon differing in energy from the energy of the incident photon by the amount of energy involved in the vibrational transition [Stolen, 1972]. Raman signals from biological molecules or cells are too weak to measure; the concern, however, arises when Raman emissions of water lie in the fluorescence emission bands. Such scattered signals are relatively weak, but since there are many water molecules ( 55 molar) in the laser beam, they can pose severe threats to flow cytometer fluorescence measurements (eg. phycoerythrin). 


\subsubsection{Excitation Sources}

Lasers are, by far, the most common sources of excitation light in flow cytometry. The main and most obvious reason is that laser beams can be tightly focused so as to put more photons through a very small area. This becomes an increasing concern when dealing with fluorescence flow cytometer systems, where increased excitation power is a necessity. Without going into a major discussion as to which lasers are best suited for certain applications, it is well known that for typical fluorescence flow cytometer systems (which use fluorescein), $488 \mathrm{~nm}$ Argon-Ion lasers are the most commonly used. Laser diodes have been proven to work for simple particle size measurements and fluorescent labels have been recently introduced commercially for red and far-red diode lasers.

Ideally, one would like to focus the laser such that all the light strikes the particles flowing down the center of the sheath. At the same time, if the laser focus is too small or too large at the laser-particle intersection, one of two things will happen: either the light will illuminate the particles locally, or not enough light will reach the particle. Thus, uniformity of light intensity is dependent on the size of the sample stream and the nature of the collection optics. Experiments performed by Loken and Stall have shown that for particle diameters of $1 \mu \mathrm{m}-30 \mu \mathrm{m}$, the ideal beam focus size is about $60 \mu \mathrm{m}$ [Shapiro, 1995]. Focusing the laser to this suggested size allows the particle to 
be sufficiently bathed with laser light without compromising intensity (see Figure 1.7).

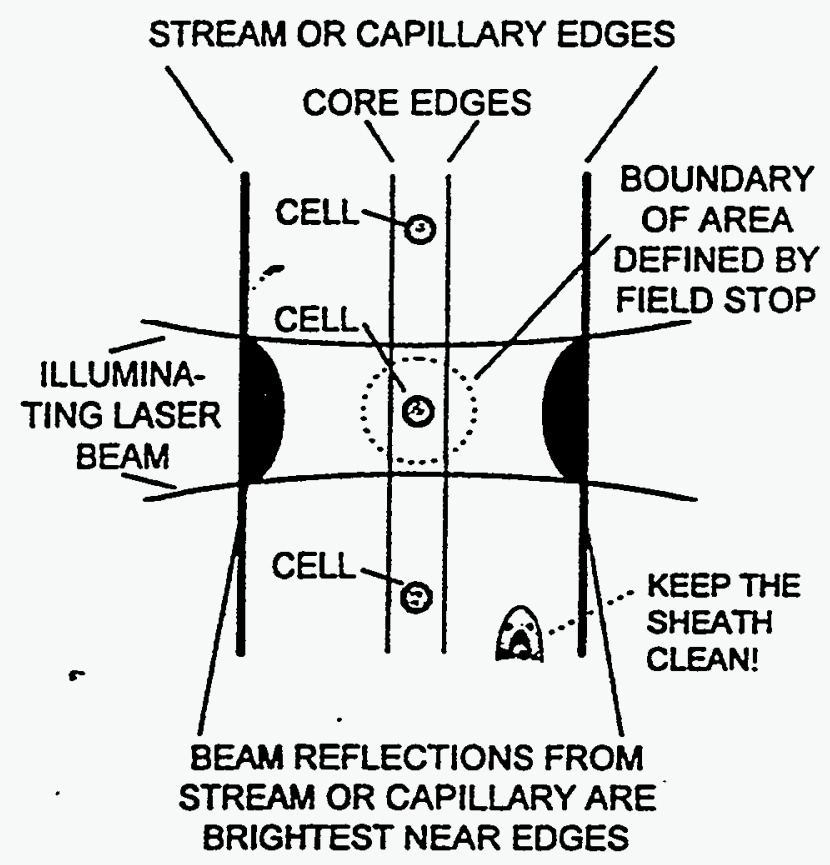

Figure 1.7- Observation point

\subsubsection{Light Collection Systems and Detection}

Successful performance of a flow cytometer hinges on the light collection and detection system. In conventional flow cytometers, light detection optics image the laser-particle intersection at 90 degrees (right angle scatter) and 0 degrees (forward scatter) from the incident light. Typical light collection systems involve quartz microscopici objectives which image and magnify the laser-particle intersection onto a light detector. Forward scatter 
optics, since they are trying to measure elastic Mie scattered light, must be spatially apertured in order to remove any reflections or refractions of the excitation beam off the stream as well as blocking the main beam. A pinhole or slit in the image plane is commonly used so that the only light which gets through is from the region of the sheath core. Right angle light collection optics, since they are primarily used for fluorescence detection, need not worry about spatial filters but can rely on interference filters, dichroics, or beam splitters to reject most of the laser line light.

Once the light collection optics have successfully captured and redirected the desired light, the light detector becomes the crucial link between experimentation and instrumentation. The two most common light detectors used in flow cytometry are silicon photodiodes and photomultiplier tubes (PMTs). Both photodiodes and PMTs produce currents when photons impinge upon them. Photodiodes are typically powered with a small bias voltage and offer little or no gain, whereas PMTs are powered with high voltage sources (sometimes up to 2000V) and offer gains which are quite high $\left(\sim 10^{7}\right)$. PMTs offer the advantage of essentially "noise-less" gain, while photodiode signals must be electronically pre-amplified. Typically, PMTs are used in fluorescence detection, while photodiodes are used for Mie scatter detection. 


\subsubsection{Flow System}

The flow system must ensure that the sample particles are channeled in a single file fashion through the laser focus. At the same time, the particles must be accurately centered $( \pm 2 \mu \mathrm{m})$ at the laser focus to guarantee consistency of scatter signal and a small coefficient of variation. The single file flow of particles is achieved using a hydrodynamic focusing flow nozzle (see Figure 1.8). Here, the sample or "core" fluid containing the particles is introduced through a narrow tube intended to be a coaxial with a wider tube through which a cell-free "sheath" fluid (usually water) is flowing. Downstream of the injection point, the nozzle diameter is reduced to channel the particles in single file fashion. Inlet pressures are regulated to prevent turbulent flows from arising.

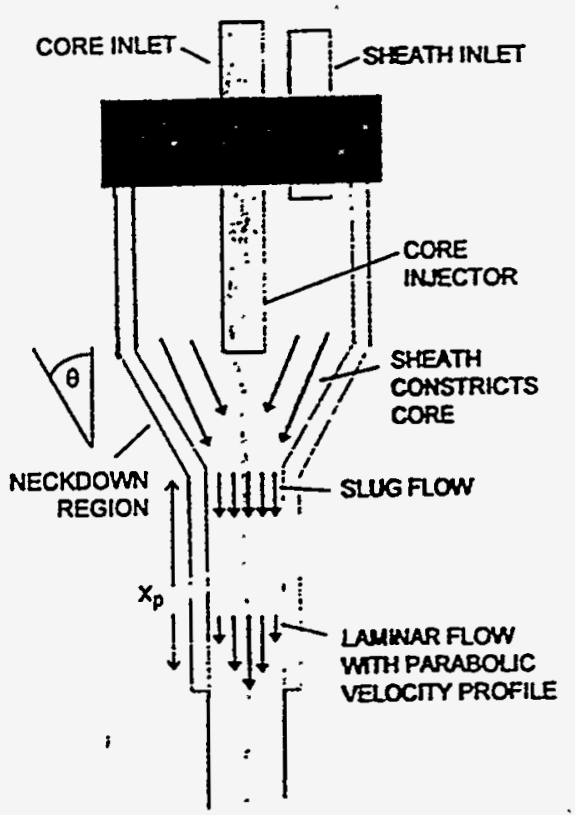

Figure 1.8 - Flow nozzle 
The observation point, at which the particles intersect the focused laser beam, varies from flow cytometer to flow cytometer. Some observe particles in cylindrical capillaries, or cuvettes with a square cross section, or through a flat window. Still others choose to view the particles in a fluid stream in air, which offers the advantage of flow-sorting. The debate over which flow system performs better has yet to be resolved.

\subsubsection{Electronics \& Signal Processing}

As has been mentioned, the outputs of photodiodes and PMTs are currents in the range of a nanoamperes to microamperes. Because some light reaches the detectors in a flow cytometer even when no particles are in the observation region, there is some output current from the detectors at all times, which can be regarded as a DC background level on which are superimposed fluctuations due to various noise sources. The passage of a particle through the observation region produces a current pulse. Information about the particle may be obtained from the peak amplitude, integral, duration, or shape of the pulse; in order to obtain this information, it is necessary to eliminate as much of the DC background and noise as possible [Watson, 1992]. 
Current pre-amplifiers convert the small current pulses into voltage pulses $(0-10 \mathrm{~V})$ which can be fed into a data acquisition system. Because the duration of these pulses are not that long $(\sim 1 \mu \mathrm{sec})$ the signal's peak amplitude must be held to give the system time to accurately sample the data. A typical flow cytometer data acquisition system will first trigger off a forward scatter signal, and then peak hold both forward and right angle scatter signals for about $10 \mu \mathrm{sec}$; after this, the system will reset and wait for another particle to pass through.

Simple electronics and signal processing systems, like the one described above, perform well when the signals are much larger than the baseline DC level. Problems arise however, when the expected signals approach the noise level of the detectors and electronics, as is the case with low level autofluorescence detection. In these situations, it is common for the data acquisition system to peak hold noise signals which are not related to the observed particle. Thus, in order to maximize the signal/noise ratio (on a signal processing level), electronic noise filters are applied at the pre-amplifier stage. In addition, since pre-amplifier gain can itself tend to be noisy, signal gain is maximized on the PMT before using the pre-amplifier. 


\subsection{The Mini-Flo Flow Cytometer}

Ray Mariella at the Lawrence Livermore National Laboratories has innovated the conventional flow stream in air flow cytometer by placing the right angle scatter (RAS) detector in the liquid itself, downstream of the observation point. Figure 1.9 illustrates the patented waveguide detection system of the Mini-Flo flow cytometer. The Mini-Flo uses the flow stream as an optical waveguide. Scattered light is captured within the flow stream and transmitted directly into a detection system. With the Mini-Flo, the conventional right angle scatter imaging/detection system is not required. Right angle scattered light is still being detected; the difference however, is that the light is orthogonal to both the horizontal plane of the light source and where the conventional RAS detector would be.

The effective numerical aperture of the Mini-Flo liquid waveguide (air/water) is 0.88 . One could place a light detector directly into the flow, with a conical tip to maintain laminar flow over the detector region. In this case, however, the area of the detector must be large enough to trap all of the rapidly diverging light from the terminus of the liquid waveguide; problems also arise with the flow stream wetting electrical contacts of the detector. Fiber optics provide a simple solution for transporting scatter signals from the liquid waveguide to the detection system. Because no commonly available fiber optic has a $0.88 \mathrm{NA}$, it is necessary to decrease the NA of the propagating 
light by expanding the beam, and for the fiber optic core to be significantly larger than the flow stream diameter. The fiber optic used in the Mini-Flo is a $220 \mu \mathrm{m}$ core multi-mode fiber with a numerical aperture (NA) of 0.44 . Since both the flow stream and fiber optic serve as multi-mode waveguides, both fluorescence and Mie scattered signals can be transmitted. The side of the fiber optic which looks into the stream is conically polished at an angle of $26^{\circ}$ to preserve the laminar flow of the stream over the core.

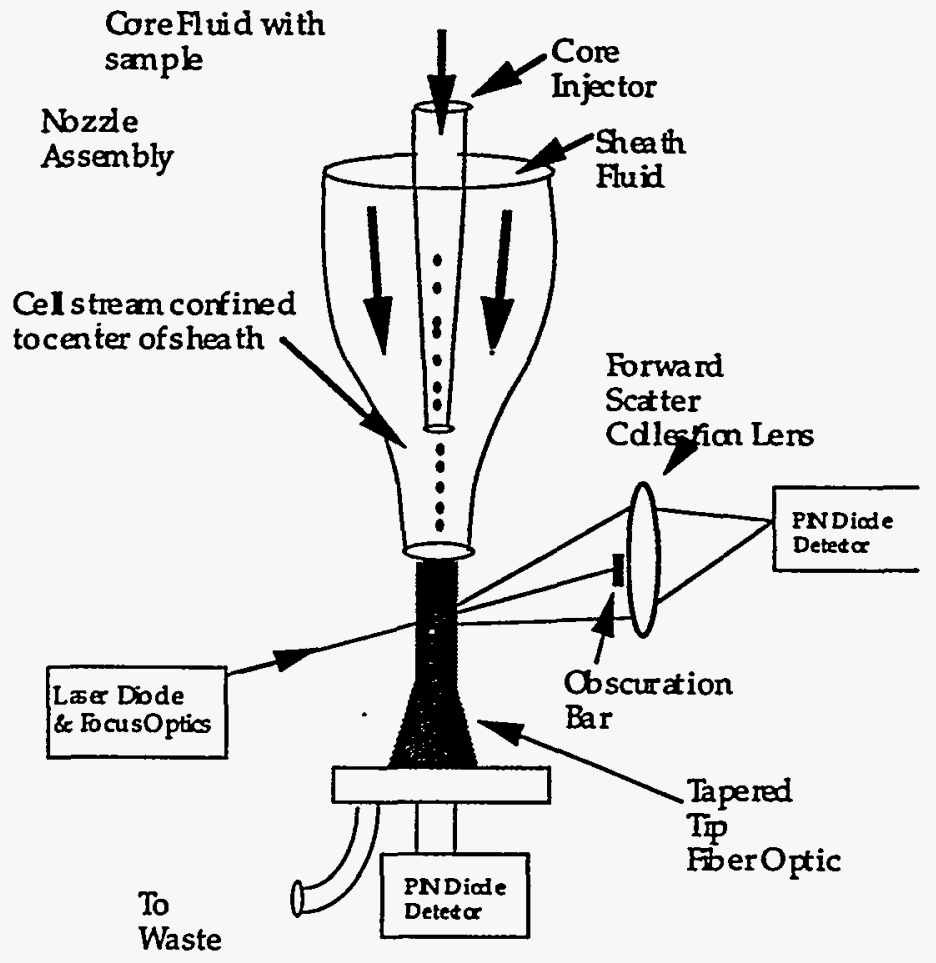

Figure 1.9 - Mini-Flo waveguide system 


\subsubsection{Mini-Flo vs. Conventional Flow Stream in Air Flow Cytometers}

The following section briefly discusses the advantages and disadvantages of the Mini-Flo over conventional flow stream in air flow cytometers.

\subsubsection{Advantages}

As mentioned before, the essential innovation of the Mini-Flo is that it provides an alternative apparatus and method for detecting scattered light in a flow cytometer. The greatest advantage of this new configuration is that it improves the signal/noise ratio for RAS light collection. As its name implies, the signal/noise ratio can be improved by either increasing the signal or decreasing the noise level; the Mini-Flo addresses both issues.

Unlike conventional flow stream in air flow cytometers, which have a very limited depth of field focus, the Mini-Flo is not an imaging system, but a guided-wave system. The main advantage is the high percent of optical throughput due to the low-loss optical waveguides. Compared to conventional flow cytometers, the Mini-Flo has far fewer surfaces for light to pass through, which directly equates to improved signal strength. 
In addition to transmitting more light, another advantage to using the Mini-Flo is the relative insensitivity to misalignments. Due to its high NA, a microscope objective has a limited depth of field and limited width of viewing plane, caused partially by apertures in the optical system. If the particles being illuminated are not at the exact focal point of the microscope objective, some of the scattered light will fail to reach the detectors and hence attenuate signal strength. The Mini-Flo, on the other hand, has no sensitivity to particle position in the flow stream, since the angle for total internal reflection is not dependent upon position. As long as the particle is close $( \pm 5 \mu \mathrm{m})$ to the laser focal point, scattered light will be transmitted through the waveguide, though the fiber optic, and into the detection scheme. The side advantages of a system that is insensitive to alignments mainly have to do with manufacturing and operating costs. Since the Mini-Flo is tolerant to $\sim \pm 5 \mu \mathrm{m}$, expensive micron positioners need not be used. In addition, Mini-Flo operators need not waste valuable sample material trying to align the system. As long as the laser is focused on the center of the flow stream, and the-flow stream falls on the tip of the polished fiber optic core, signal strength will not be compromised.

As mentioned before, signal/noise ratio improvements are also achieved by reducing the system's background level. In conventional flow cytometers, a major source of background comes from the reflections of incident light on the outer flow stream surface. In forward scatter optics, a 
majority of this light is spatially apertured out; some light, however, still manages to reach the detector through secondary reflections and other Mie scattering processes that, to this date, are still not completely understood. In the Mini-Flo, just as the waveguide traps light inside through total internal reflection, it also rejects a majority of outside light through the same process. Incident light reflecting off the flow stream surface simply cannot enter the waveguide and therefore the background levels in the Mini-Flo are considerably less.

Some other advantages that go beyond the theoretical discussion of the Mini-Flo are worth mentioning. First, because it eliminates any need for microscope imaging systems, the Mini-Flo has potential to become the smallest flow cytometer in the world. This ties into the prospect of putting flow cytometry on a chip, through microscopic etching (the only limiting issue of course being containment of the water, which cannot be miniaturized). Second, and probably the most important advantage of this system, is its low manufacturing cost. The main expenses that come from building a flow cytometer system are the laser and the expensive precision optic systems. While the Mini-Flo needs a laser source as well, the need for precision microscope objective lenses are not. Preliminary investigations rate the manufacturing costs of the Mini-Flo to be about $\$ 10,000$, compared to the commercially available flow cytometers which start at $\$ 80,000$. 


\subsubsection{Disadvantages}

The main disadvantage to having a guided-wave system as opposed to an imaging system lies in the resolution of the signal. While the signal strength is greater in the Mini-Flo, resolution of the signal is compromised. In most cases, this does not present a serious issue; however, there are certain applications where signal resolution can give important and valuable information pertaining to particle morphology. Practical application of the Mini-Flo are discussed at a later point.

Another disadvantage, about which not much is known about, is natural fluorescence of the fiber optic. Howard Shapiro and Michael Hercher briefly explored this issue and quickly concluded that a flow cytometry system using fiber optics would be difficult to build primarily because of fiber optic auto-fluorescence, which can easily mask true fluorescence signals [Shapiro, 1986]. This is one of the main issues addressed in later sections. Similar to fiber optic fluorescence, the mechanical dynamics of a flow stream in air has become a recent topic of study. Although it has not been investigated in depth, minute surface oscillations. on the flow stream surface can possibly cause low level noise signals (experienced only in flow stream in air flow cytometers, and not cuvette style systems). This problem, however, requires extensive computer modeling and extends beyond the scope of this thesis. 


\subsubsection{Mini-Flo \& Possible Applications}

Because of its ease of portability and cost effective light collection system, Mini-Flo has a number of applications in the biological detection field. Mini-Flo technology can be used for detection of hazardous biological bacteria or viruses. In addition to these, the Mini-Flo is useful for simple particle sizing.

As stated before, the goal of this thesis was to perform an in depth study of all sources of background in the Mini-Flo and suggest what can be done to improve the signal/noise ratio. The remainder of this thesis discusses the results and conclusions of this study. In addition, some time is spent proposing a new design for the Mini-Flo, and suggesting further studies to improve the Mini-Flo's performance. 


\section{- 2.0 The Mini-Flo: Detailed Design Discussion}

Before explaining the experiments performed on the Mini-Flo, it is essential to understand the current design. Figure 2.1 shows a picture of the Mini-Flo. The Mini-Flo is a one fluorescence channel dedicated system for fluorescein. The flow chamber area consists of a flow nozzle, which directs a $50 \mu \mathrm{m}$ stream directly onto the fiber optic tip. The fiber optic is mounted within a continuously pumped cup which removes all the used sheath and sample fluid from the chamber. The fiber optic is polished to a conical angle of $26^{\circ}$ on one end, and terminates into an optics package on the other. From Figure 1.6, fluorescein emission occurs between 510 and 550 nanometers, while excitation absorption is maximum at $485 \mathrm{~nm}$ (due to limited resources, the excitation laser source was at $476.5 \mathrm{~nm}$ ). The optics package, therefore, must separate and detect scattered light and fluorescence emissions. The optics package first consists of a collimating lens. The light is then sent through a dichroic mirror which reflects RAS light and transmits fluorescence. The reflected RAS light is sent through a 500nm low pass filter and is then focused onto a silicon photodiode. The transmitted fluorescence light is first sent through two filters: a Chroma $535 \mathrm{~nm} \pm 25 \mathrm{~nm}$ bandpass filter and a Chroma 500nm high pass filter; this light is then fed into a PMT (see Figure 2.2). 


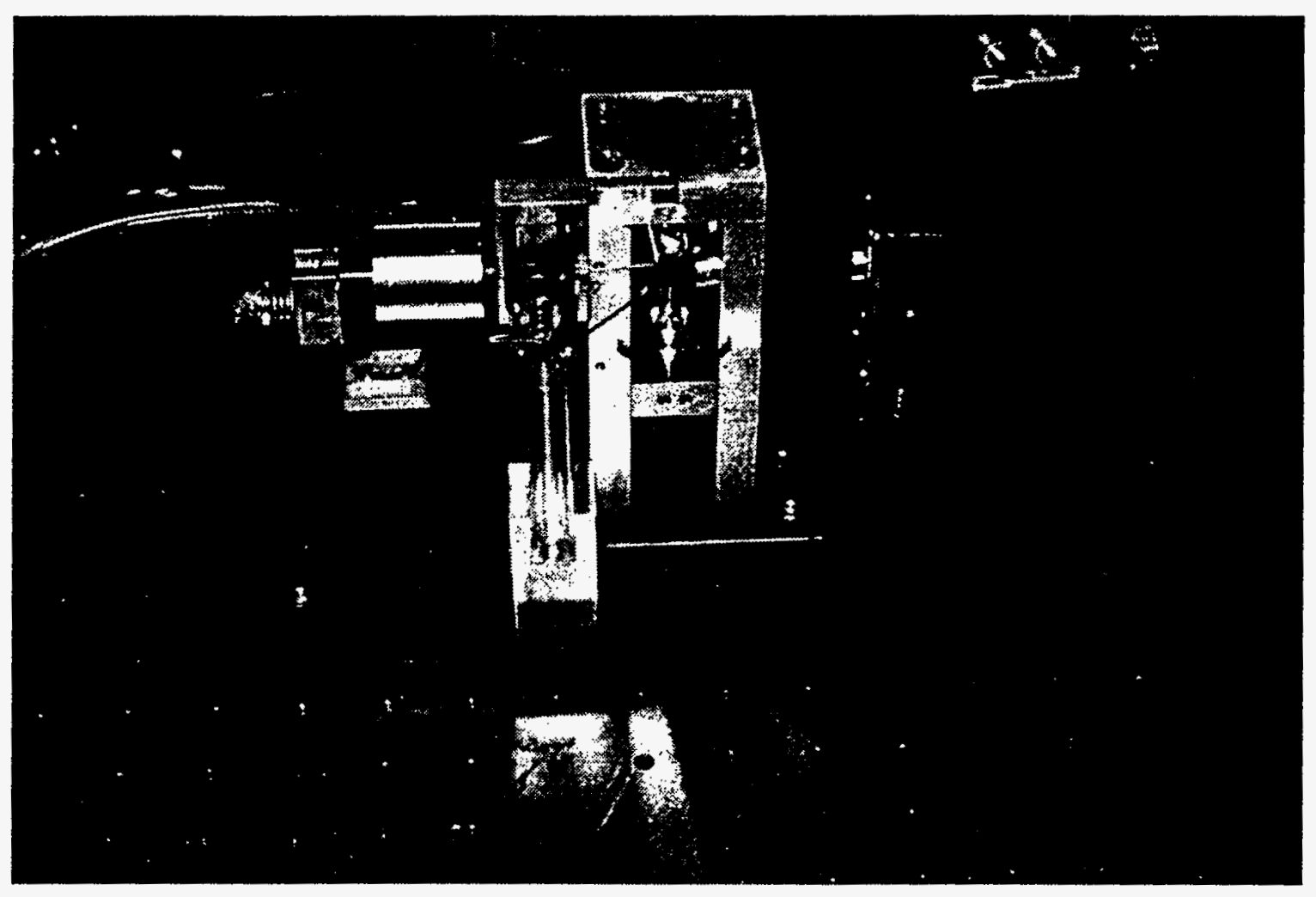

Figure 2.1 - The Mini-Flo flow cytometer

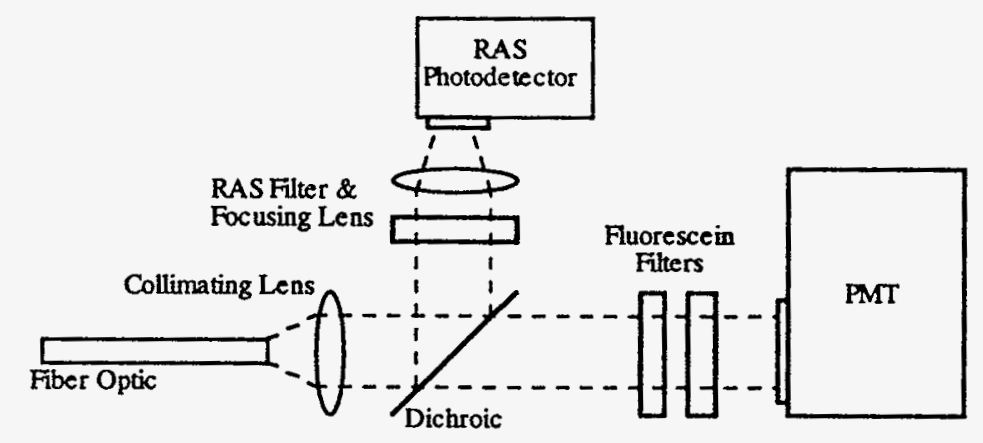

Figure 2.2 - Optics package schematic 


\subsection{Signal/Noise Ratio Issues}

Signal/background ratio issues become significant when distinguishing between weakly fluorescent and non-fluorescent particles. Presently, weakly fluorescent particles create a $500 \mathrm{mV}$ signal on the PMT, while nonfluorescent bead signals get lost in background levels of $\sim 60 \mathrm{mV}$. Thus, due to the background levels, the factor of difference between fluorescent and nonfluorescent particles is only about 8; the desired performance of the Mini-Flo

.. is a difference factor of about 100. The following sections discuss possible sources of noise and what can be done to remove them.

\subsection{PMT Performance}

In order to detect weakly fluorescence signals, high voltage gains are required on the PMT. At high voltage gains however, spontaneous emissions of single electrons which are not light dependent occur. These emissions create signals $(\sim 100 \mathrm{mV})$ which are much like fluorescence signals (see Figure 3.1). There are two possible ways of eliminating such a noise source: (1) use a PMT with very low dark current levels, or (2) spectrally filter out the noise. PMTs with no dark current (or at least very little) can be achieved by cooling the PMT housing; spontaneous emissions become less frequent as the 
temperature drops. Electronic noise filtering solves the problem as long as the frequency of the noise does not coincide with the frequency of the data.

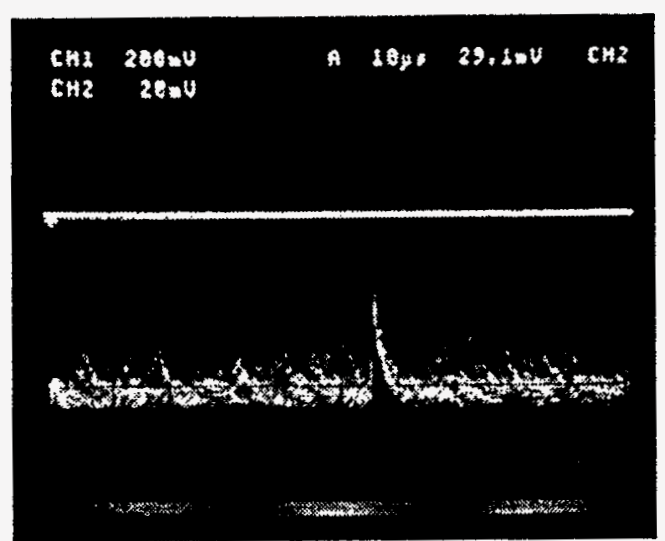

Dark Current Signals

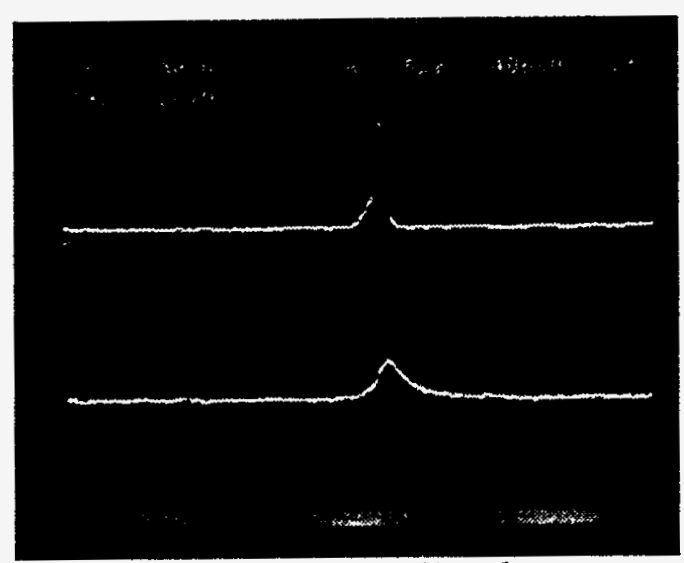

Fluorescence Signals

Figure 3.1 - Dark current signals vs. Fluorescence signals

At the same time, since the gain is very high, the PMT is extremely sensitive to any light, even single photons. Thus, optical leakage is a crucial part of the background level problem.

\subsection{Lenses, Filters, \& Fiber Optics}

In an ideal world, with perfect optics and coupling, only fluorescence light emanating from the particle would reach the PMT. Unfortunately, in the real world, partial transmission, poor optical coupling, and autofluorescence play significant roles. As pointed out before, at high PMT gain 
levels, single photons (from non-sample fluorescence, chamber walls, optics, etc.) contribute greatly to the background level.

\subsubsection{Optical Leakage}

Even though a dichroic mirror and two filters are placed before the PMT, optical leakage of $476.5 \mathrm{~nm}$ light is possible. This is mainly due to the transmission characteristics of the optics. The dichroic mirror is designed to reflect $98 \%$ of $476.5 \mathrm{~nm}$ light, thereby allowing. $2 \%$ to pass through (see Figure 3.2). The Chroma $535 \mathrm{~nm} \pm 25 \mathrm{~nm}$ bandpass and $500 \mathrm{~nm}$ high pass filters have $0.3 \%$ and $1 \%$ transmission characteristics for $476.5 \mathrm{~nm}$ light respectively. Thus, out of $100 \%$ of blue light which enters the optical package, approximately $6 \times 10^{-5} \%$ reaches the PMT. At very high PMT gain levels, this seemingly low amount of light can cause significant background levels. This background source cannot be eradicated; however, by placing more filters before the PMT, less blue light.will be transmitted. At the same time though, fluorescence signals will be attenuated because these filters typically transmit about $95 \%$ of the actual light. 


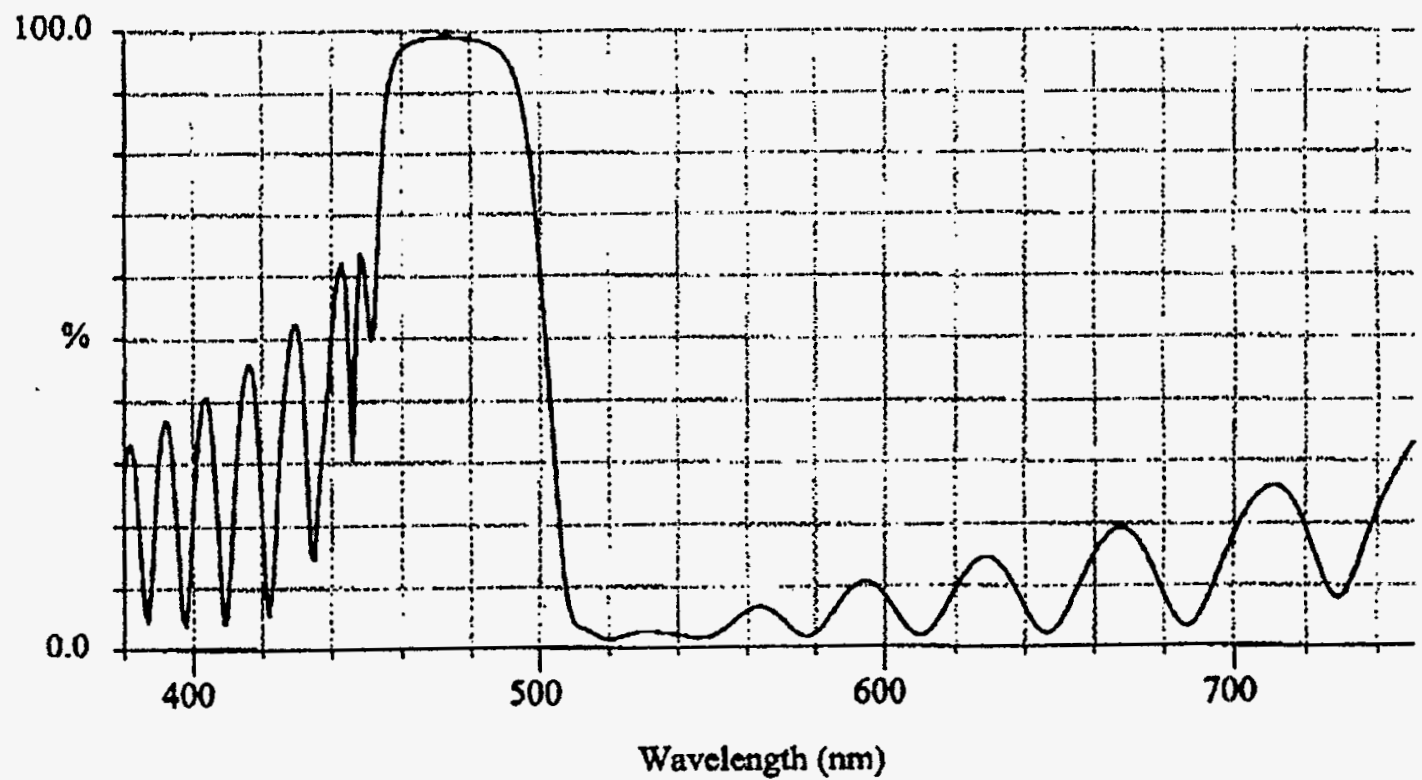

Figure 3.2 - Percent reflectivity characteristic for dichroic

\subsubsection{Optic Auto-Fluorescence}

Lenses, filters, and fiber optics have all been known to fluoresce at $530 \mathrm{~nm}$ when struck with light between $450 \mathrm{~nm}$ and $500 \mathrm{~nm}$. This phenomenon poses a serious background problem to the Mini-Flo, because scattered light induced by non-fluorescent particles can create background fluorescence signals when they strike various optical surfaces. Glass lenses and fiber optics can be replaced by the less fluorescing (but more expensive) UV-grade quartz material. The problem may be bypassed by characterizing the fluorescence capability of each lens, and predicting what is the overall noise 
level due to auto-fluorescence. Once this is known, post-processing of the data can eliminate the noise level to reveal the true fluorescence signal size.

\subsection{Raman Scattering}

Inelastic Raman scattering poses a problem when the light emitted lies in the fluorescence detection range. For the Mini-Flo, Raman scattering of water sheath occurs at about $580 \mathrm{~nm}$. This light falls out of the fluorescein emission range and therefore is not a significant noise issue. Had the MiniFlo been designed to detect phycoerythrin which fluoresces between $570 \mathrm{~nm}$ and $620 \mathrm{~nm}$, Raman water scattering would have presented a problem.

\subsection{Miscellaneous Noise Sources}

There are a number of other possibilities that can create noise in the Mini-Flo system. For example, random particles in the flow stream can create scatter signals and, at times, very weak fluorescence signals. In order to avoid this, all water flowing through the system should be filtered, and everything in general should be kept clean. 
Ambient light from the environment can easily leak in by way of poor optical shielding. Since most environment light consists of all visible wavelengths, $530 \mathrm{~nm}$ light can pass through all the way to the PMT. Thus, it is absolutely vital to keep the Mini-Flo optics system in darkness. Depending on the flow over the tip, environmental light has also been known to enter through the fiber optic in the flow chamber. Therefore, it is also a good idea to keep the flow chamber dark.

Finally, instrumentation noise can create signals which have no relation to the optics or the flow. Ground loops, radio frequency noise, and power supply noise have all been known to cause low level phantom RAS and fluorescence signals. Hence, it is important to keep the entire Mini-Flo system in static free environment. 


\subsection{Experimental Process \& Results}

The following section details the experiments performed on the MiniFlo regarding the signal/noise ratio issues discussed above.

\subsection{Spectral Analysis}

Spectral analysis of the Mini-Flo was performed to confirm fluorescein emission between 500 and 550 nanometers and Raman scattering of water at a narrow bandwidth around 580nm. A DigiKröme monochromater was first coupled to the fiber optic end, and then attached to a PMT; spectral scans were taken at various states of Mini-Flo operation. First, Polyscience fluorescent beads were used to determine the actual fluorescein emission bandwidth. A $500 \mathrm{~nm}$ long pass filter was placed between the monochromater and the PMT to ensure rejection of any and all "ghost" reflections of $476.5 \mathrm{~nm}$ light. Light intensities measured on the PMT were converted to voltage pulses through current pre-amplifiers, and then recorded on a data acquisition system. Wavelength scans were taken from 500 to 650 nanometers, while Polyscience fluorescent beads ran through the system (see Figure 4.1). The PMT voltage gain during this experiment was set at $640 \mathrm{~V}$ and the laser power was set at $150 \mathrm{~mW}$. Various size slit apertures were used to gauge the difference between signal strength and resolution. As expected, the fluorescence emission range 
lied between 500 and 550 nanometers; Raman scatter signals from the water could not be detected during this experiment because the voltage gain on the PMT was too low.

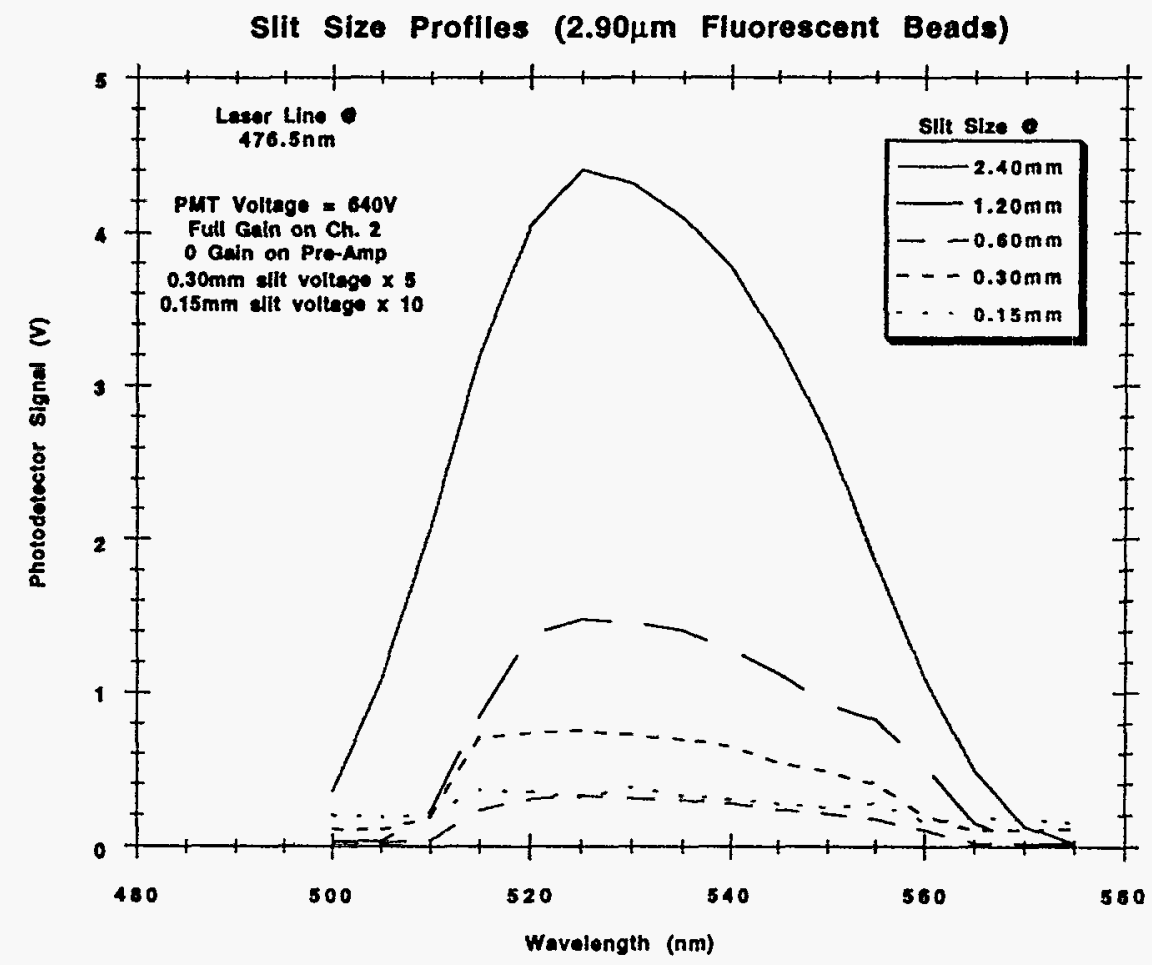

Figure 4.1 - Wavelength scans for Polyscience beads

Raman water scatter signals were analyzed by running only water (no sample) through the Mini-Flo and raising the PMT gain to its maximum voltage $(1250 \mathrm{~V})$. As before, a $500 \mathrm{~nm}$ long pass filter was used to prevent laser light from reaching the PMT. Wavelength scans (between 500 and 650 nanometers) were first performed for $476.5 \mathrm{~nm}$ laser light. Once a Raman signal was recorded, the same experiment was performed for $496.5 \mathrm{~nm}$ and 
$514 \mathrm{~nm}$ laser light. Due to the shift in wavelengths, a corresponding shift in Raman peaks was expected, as illustrated in Figure 4.2.

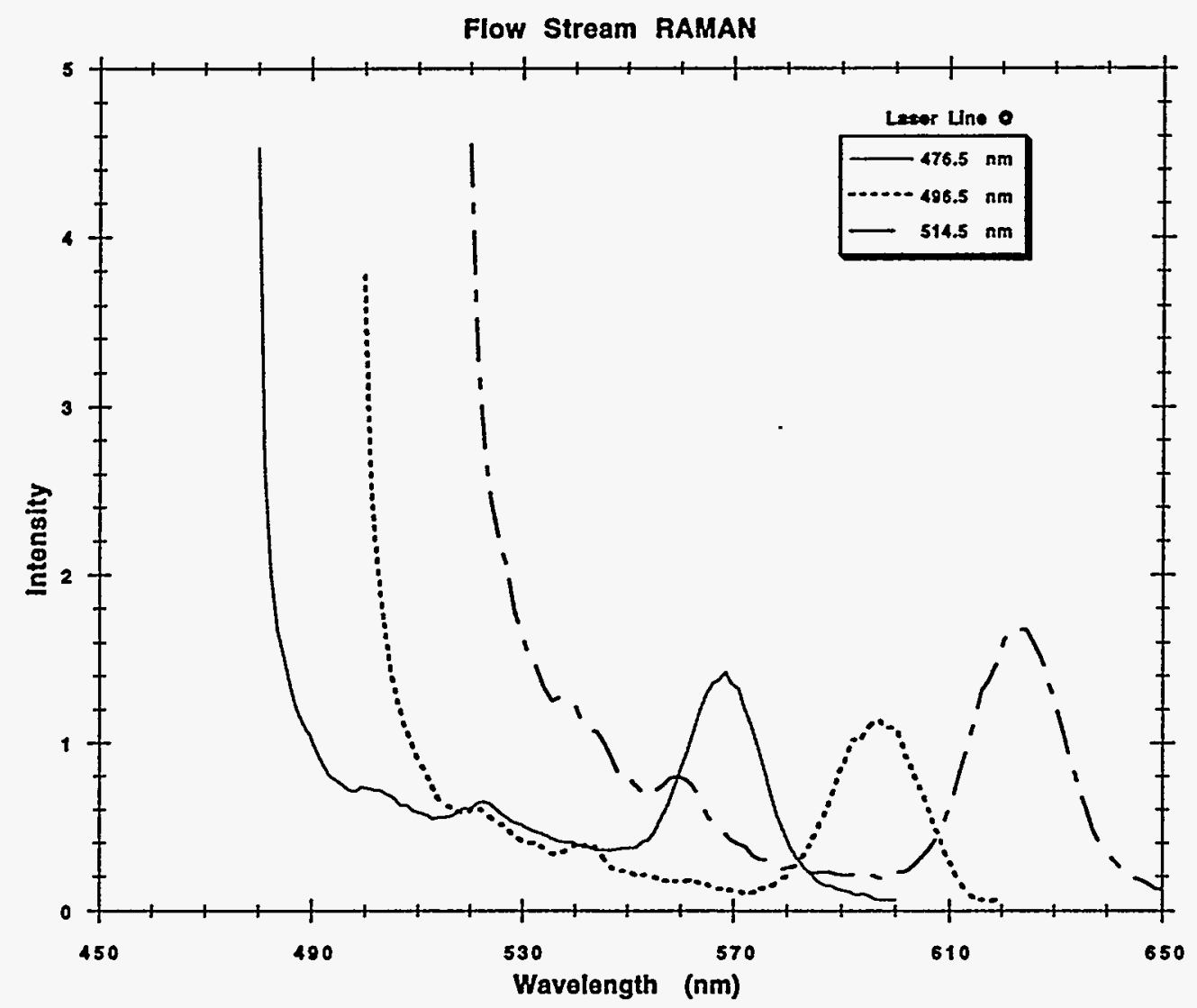

Figure 4.2- Wavelength scans for Raman scattering of water

\subsection{PMT Noise}

Before investigating low level noises such as optical auto-fluorescence, the inherent background noise level of the PMT had to be determined. This was achieved through a series of investigations with the PMT set at full gain (1250V). First, the pure "dark current" signal was measured; PMT signals were then measured when the laser was on, and then with the water flowing. Due 
to the low levels of voltage in these investigations, the data acquisition system (which has a resolution of $50 \mathrm{mV}$ ) could not be used. Figures 4.3, 4.4, and 4.5 are photographic images of the oscilloscope taken during each PMT experiment. Channel 1 (upper line) reads the side scatter elastic signals, while Channel 2 (lower line) reads the inelastic fluorescence signals. From the figures, the "dark current" voltage signal was approximately $10 \mathrm{mV}$; with the laser on, spontaneous $20 \mathrm{mV}$ peaks would appear and when the water was turned on, the noise immediately jumped to about $40 \mathrm{mV}$.

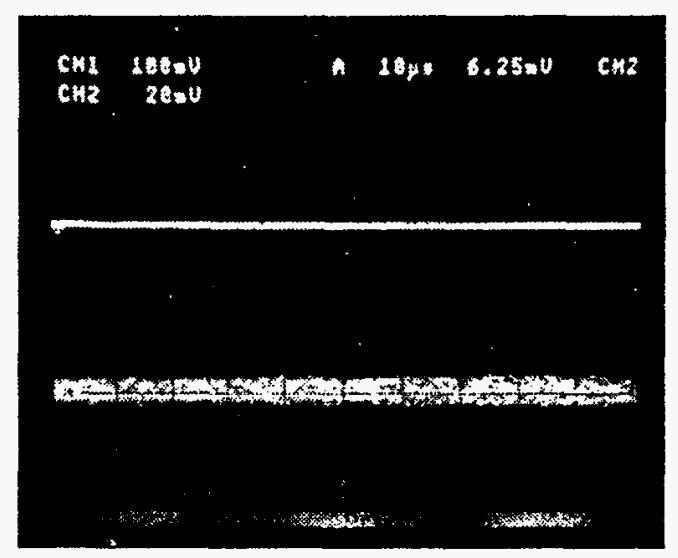

Figure 4.3 - PMT dark current photo

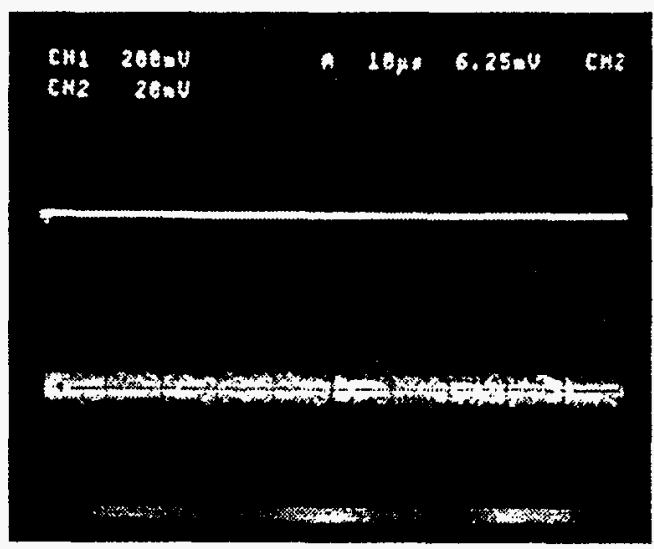

Figure 4.4 - PMT with laser on 


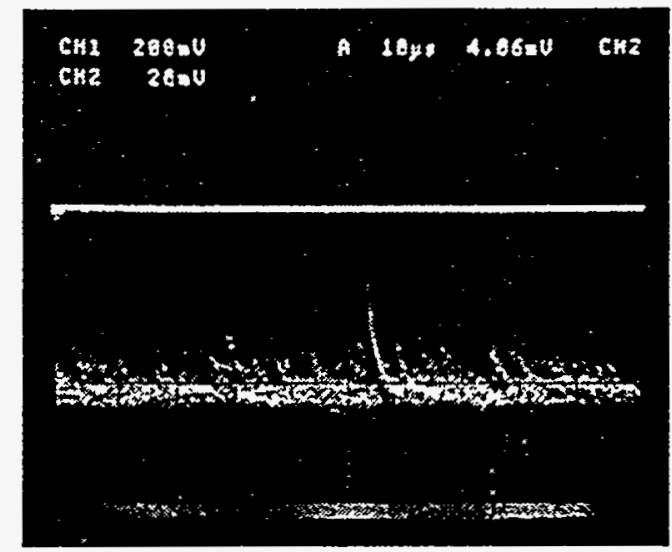

Figure 4.5 - PMT with water and laser on

Ideally, since it only looks for fluorescence (and no fluorescence is expected), Channel 2 would have remained at $10 \mathrm{mV}$ when both the laser and water were turned on. However, since the noise level did increase significantly, there are three possible explanations: (1) laser light source is impure and emitting light at $\sim 530 \mathrm{~nm}$ as well, (2) blue light is leaking through the optics. and reaching the PMT, or (3) optical surfaces including the fiber optic are auto-fluorescing at $530 \mathrm{~nm}$.

\section{3 laser Purity \& Optical Leakage}

In order to ensure laser purity, a 480/40 nm bandpass filter was placed in front of the laser before the Mini-Flo. It should be noted however, that while some impurities were observed in the laser beam, placing the 
480/40nm filter made little or no difference in the PMT noise discussed above.

As discussed before, according to Figure 3.2, the dichroic beam splitter transmits about $2 \%$ of $476.5 \mathrm{~nm}$ light and reflects the rest. With the incorporation of the $535 / 50 \mathrm{~nm}$ bandpass filter between the dichroic and the PMT, $6 \times 10^{-3} \%$ of $476.5 \mathrm{~nm}$ light reaches the PMT. In order to confirm these transmission characteristics experimentally, Polyscience fluorescent and nonfluorescent beads were run through the Mini-Flo with two different optical set-ups: (1) dichroic followed by the 530/50nm bandpass filter, and (2) dichroic followed by a $510 \mathrm{~nm}$ long pass filter and then the $530 / 50 \mathrm{~nm}$ bandpass filter. PMT voltage gains were set at $550 \mathrm{~V}$ and $1250 \mathrm{~V}$ for the fluorescent and nonfluorescent beads, respectively. Figures 4.6 and 4.7 show the oscilloscope signals of both beads for the first and second optical set-ups. With the addition of the 510nm long pass filter, fluorescence intensities for both beads were reduced by $\sim 75 \%$. There are two possible explanations for this significant drop in light intensity: (1) optical leakage of blue light in the first set-up or (2) autofluorescence of the $530 / 50 \mathrm{~nm}$ filter. Both are viable explanations; determining which one is more prevalent requires detailed spectral analysis of each optical component (which extends beyond the scope of this thesis). It therefore suffices to say that once the 510nm long pass filter was added, blue light that would have either passed through the single $530 / 50 \mathrm{~nm}$ filter or caused it to fluoresce was no longer allowed. Incidentally further additions of 
510 long pass filters reduce intensities by only $\sim 10 \%$, which can probably be attributed to light absorption of the extra material (see Figure 4.8). In this way, it is possible to place enough filters so that the signal of non-fluorescent beads is reduced to near zero. At the same time however, the true fluorescence signals of the fluorescent beads will also be significantly reduced.

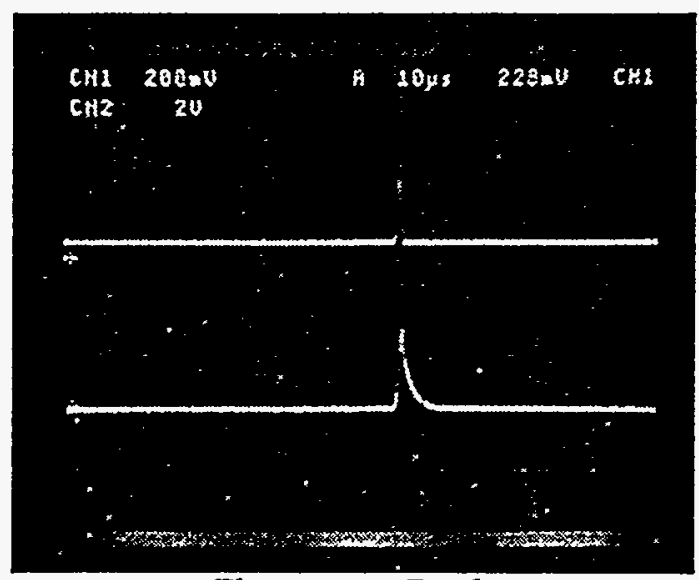

Fluorescent Beads

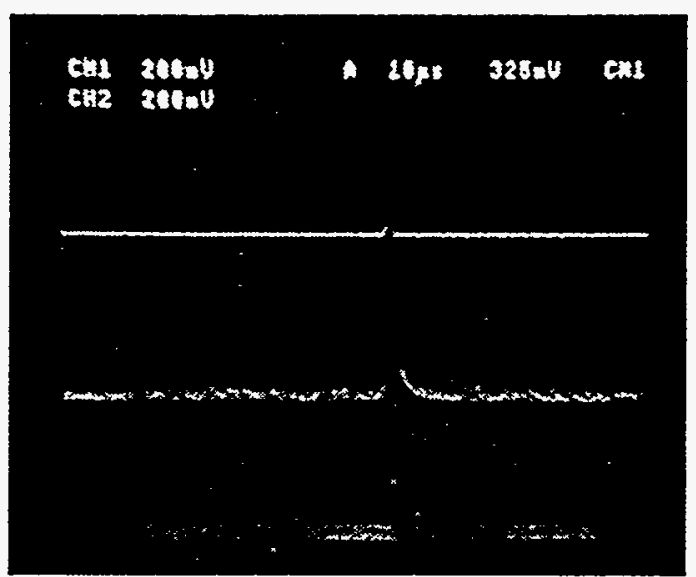

Non-fluorescent Beads

Figure 4.6 - Oscilloscope signals of Polyscience fluorescent and non-fluorescent beads with Chroma $530 / 50 \mathrm{~nm}$ bandpass filter

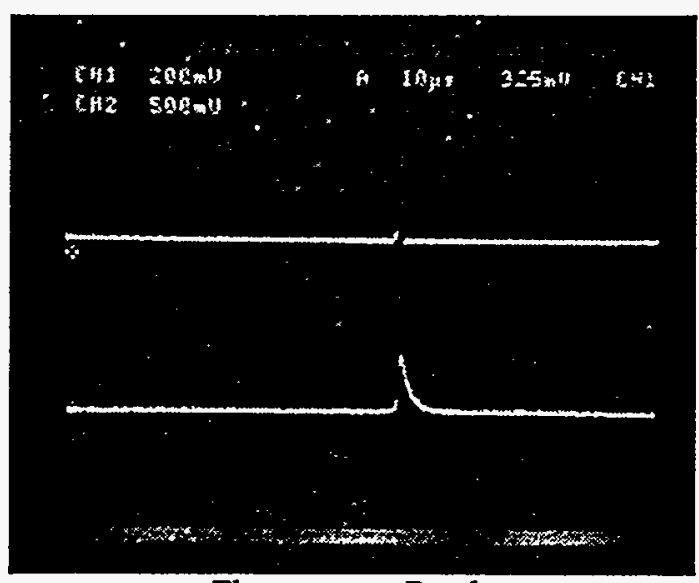

Fluorescent Beads

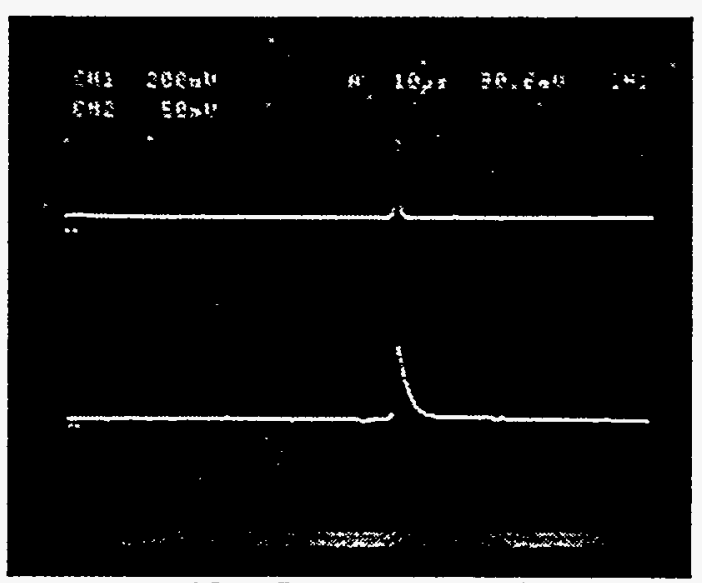

Non-fluorescent Beads

Figure 4.7 - Oscilloscope signals of Polyscience fluorescent and non-fluorescent beads with Chroma 530/50 $\mathrm{nm}$ bandpass and $510 \mathrm{~nm}$ longpass filter 


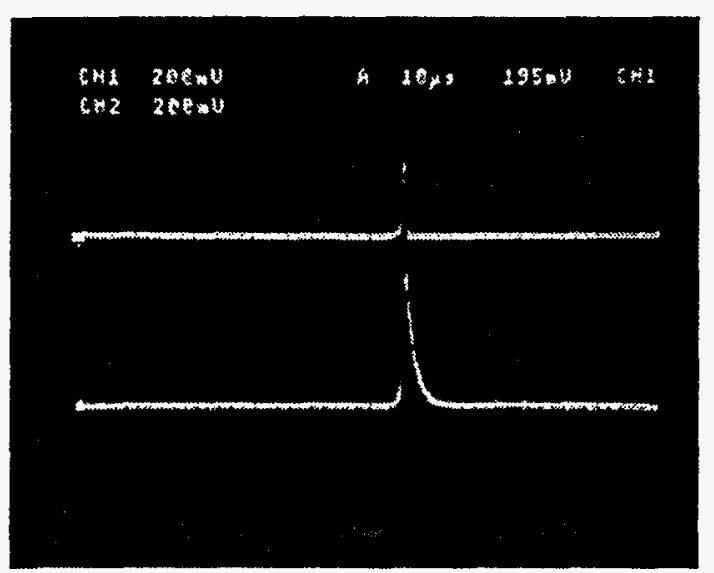

Fluorescent Beads

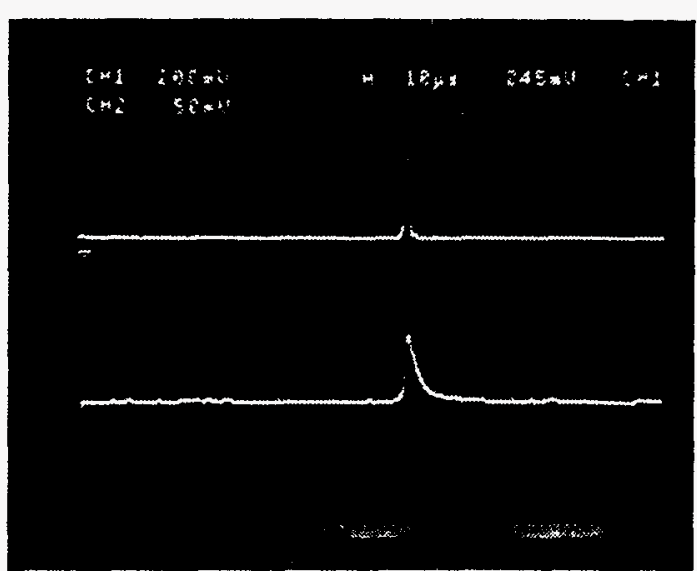

Non-fluorescent Beads

Figure 4.8 - Oscilloscope signals of Polyscience fluorescent

and non-fluorescent beads with Chroma 530/50 $\mathrm{nm}$ bandpass and two $510 \mathrm{~nm}$ longpass filters

Although optical leakage is a significant noise issue, it still does not explain why a non-fluorescent bead produces any fluorescence signal. Many "non-fluorescent" beads are known to have low levels of auto-fluorescence. However, these Polyscience non-fluorescent beads were tested on a FacScan machine and determined to have absolutely no fluorescence. Therefore, the only other possibility of noise lies in optical auto-fluorescence.

\subsection{Optical \& Fiber Optic Auto-Fluorescence}

All optical surfaces in the Mini-Flo are capable of creating low level fluorescence signals when struck with blue light. Therefore, when elastically side scattered light pulses move through the optics package, phantom fluorescence signals are created as well. Considering all optical surfaces (except 
the fiber optic), those surfaces closest to the PMT have the most significant impact; thus, attention must be focused on four surfaces, the collimating lens, the dichroic mirror, the 510 long pass filter, and the $530 / 50$ bandpass filter. The lens which focuses the laser to the intersection point, while it might fluoresce, has little or no effect since it is not directly coupled to the PMT. As mentioned before, a detailed spectral analysis of each optical component would extend beyond the scope of this thesis. However, some quick studies were performed to gauge the importance of this issue.

Assuming the fiber optic does not fluoresce, the light coming from there is real and properly collimated by the collimating lens. Autofluorescence light generated at the various optical surfaces, on the other hand, will radiate in $2 \pi$ steradians from the source (see figure 4.9 ). Therefore, if the PMT is placed further away from the optical surfaces, the real collimated light signals will reach the PMT with little attenuation, while the autofluorescence light will be quickly reduced as the distance is increased. Signals were compared for two different optical configurations. The first configuration placed the PMT one inch away from the first optical surface, while the second configuration placed it three inches away. Figures 4.10 and 4.11 show the oscilloscope signal sizes for the two configurations; there is no significant difference between the two, indicating that auto-fluorescence of the collimating lens, dichroic mirror, and filters is not a significant issue, and attention must be focused to the fiber optic. 


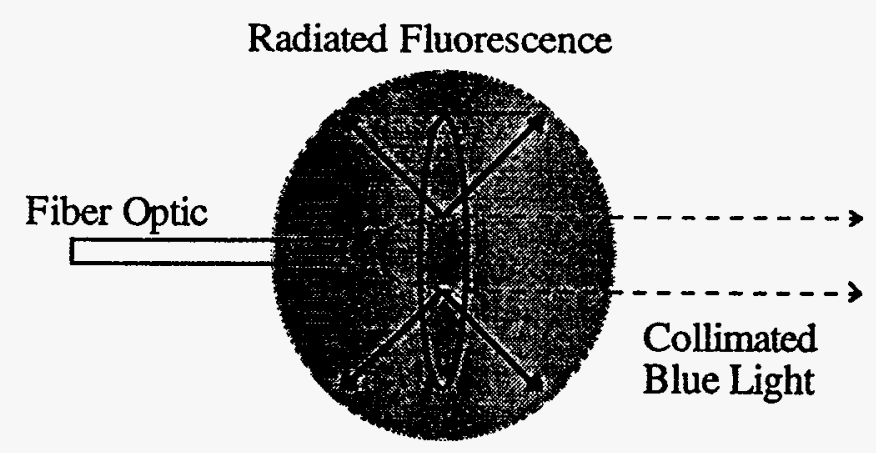

Figure 4.9 - Illustration of filter auto-fluorescence

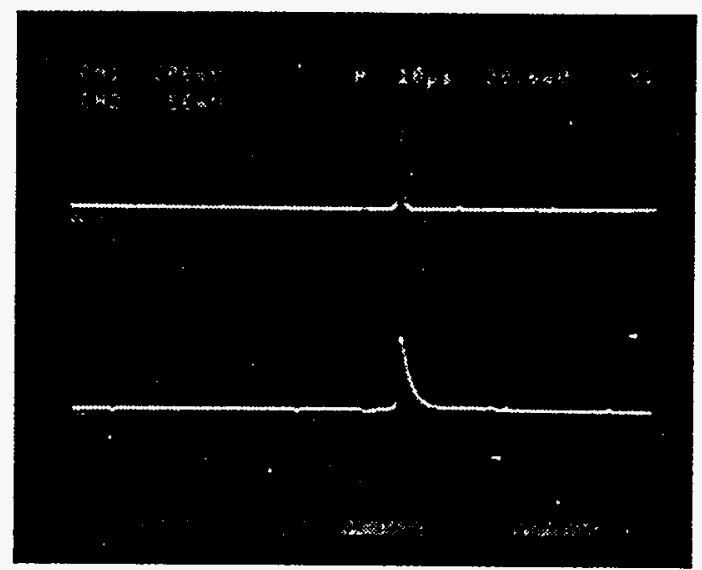

Figure 4.10 - Oscilloscope signals of Polyscience non-fluorescent beads with at 1" distance

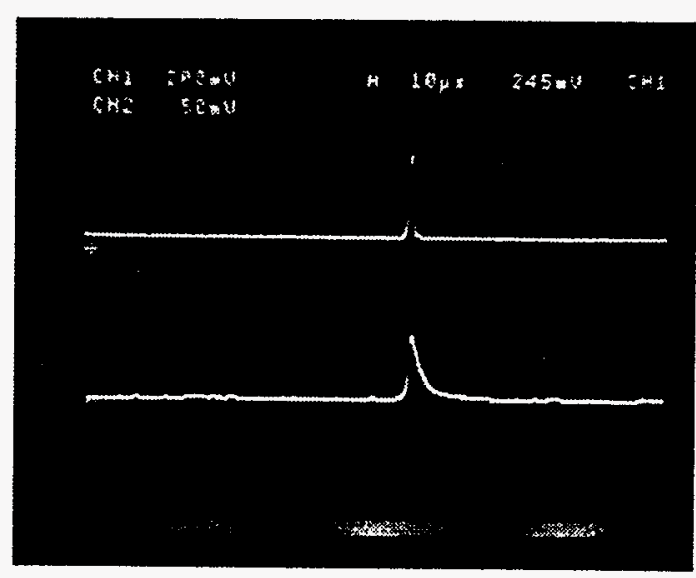

Figure 4.11 - Oscilloscope signals of Polyscience non-fluorescent beads with at $3^{\prime \prime}$ distance 
Fiber optic fluorescence poses a serious problem because "phantom" fluorescence signals will be collimated and transmitted to the PMT. Since fluorescence levels of the fiber optic are dependent on volume of material (and therefore length), a comparison was made between a fiber optic of $13^{\prime \prime}$ and one of $3 "$. FCS fluorescent and Polyscience non-fluorescent beads were also run with this set-up and compared to runs with a fiber length of 13" (see figures 4.12 and 4.13). As we expect, since the length was reduced by a factor of $\sim 4$, the fluorescence noise levels decreased to an almost undetectable level. With the shorter fiber, the fluorescence levels decreased for both beads; assuming non-fluorescence signals were strictly noise in the fluorescence channel, the signal/noise ratio increased from $\sim 25$ to $\sim 75$.

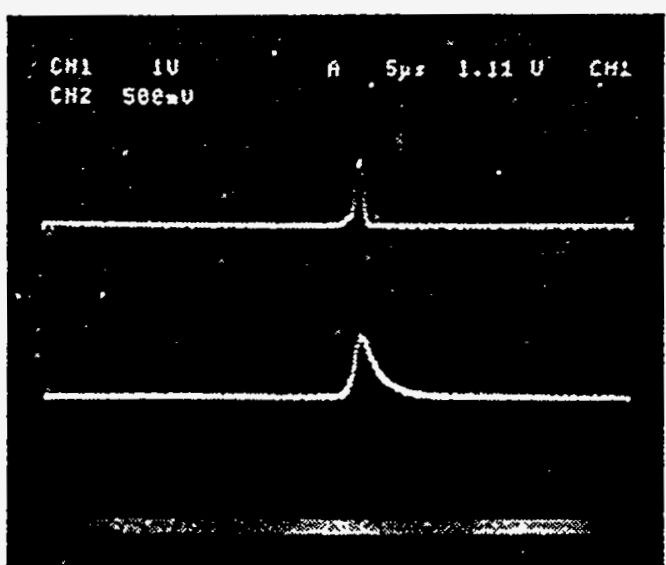

FCS Fluorescent Beads

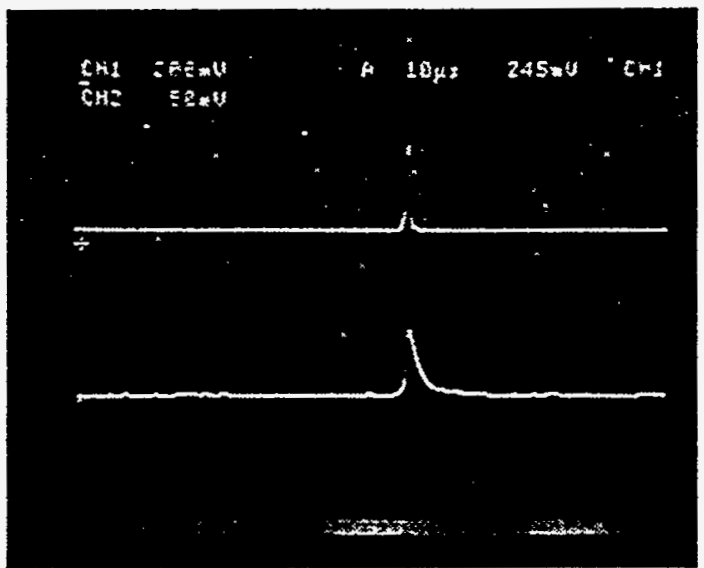

Polyscience Non-fluorescent Beads

Figure 4.12 - Oscilloscope signals of FCS fluorescent and Polyscience non-fluorescent beads with $13^{\prime \prime}$ fiber optic 


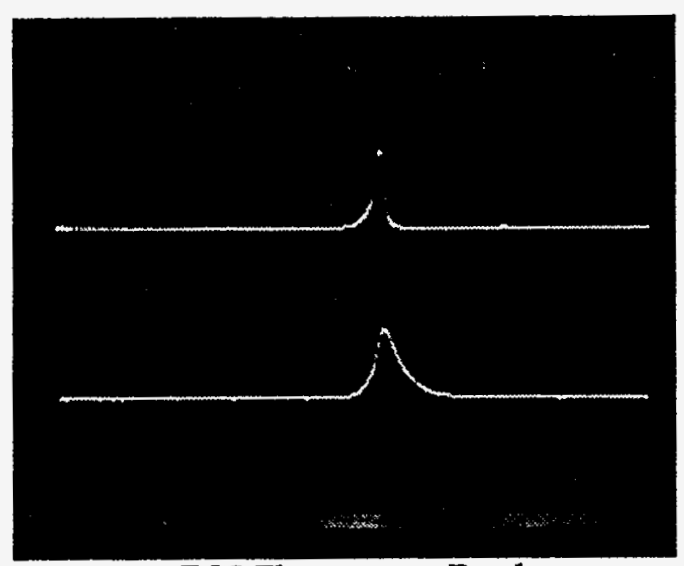

FCS Fluorescent Beads

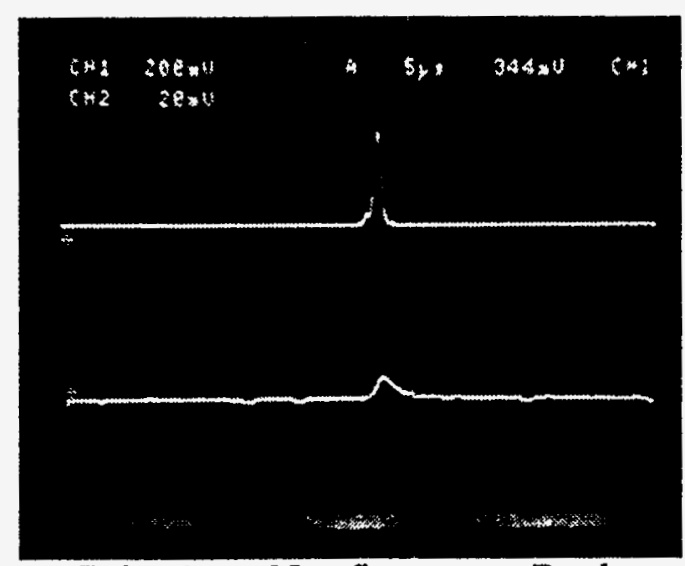

Polyscience Non-fluorescent Beads

Figure 4.13 - Oscilloscope signals of FCS fluorescent and Polyscience non-fluorescent beads with 3 " fiber optic

A spectral analysis of the fluorescence of the fiber optic at $476.5 \mathrm{~nm}$ light was also performed to confirm the nature of its fluorescence. $476.5 \mathrm{~nm}$ light was sent through one end of the fiber optic and the output was spectrally analyzed. In order to simulate the levels of $476.5 \mathrm{~nm}$ light entering the fiber optic during a real experiment, blue elastically side scattered light pulse levels were first recorded with Polyscience non-fluorescent beads running. Once this light level was determined, an integrating sphere was used and the light intensity adjusted accordingly. Out of $100 \%$ blue light which entered the fiber optic, $30 \%$ was converted to $530 \mathrm{~nm}$ light. This confirms the notion that the fiber optic fluoresces and elimination or reduction of this can significantly increase the signal/noise ratio. 
Unfortunately, there is no quick solution to this problem; the fiber optic is integral to the Mini-Flo and cannot be removed or replaced. Fiber length can be minimized as shown above, but the noise will still exist, and not be completely eliminated. Different fibers exist which have lower fluorescence levels, such as Suprasil; these fibers however, are more expensive, and must be special ordered for large numerical apertures of 0.44 or core sizes of $220 \mu \mathrm{m}$. Knowing that the fiber optic is the source of noise, one may endeavor to quantify the fluorescence noise level for each bead size being used, and then eliminate it in the post-processing stage. Different bead sizes scatter different amounts of light into the fiber optic and therefore, this can become a painstaking and possibly ineffective process.

\subsection{Other Possibilities}

The following sections address side issues which could have also affected the signal/noise ratio.

\subsubsection{Random Particles in Sheath}

In order to guard against micron size particles from entering the flow chamber, the sheath water was first distilled and then run through a $0.22 \mu \mathrm{m}$ filter. Special care was taken to keep everything clean; proper ventilation and 
circulation in the room was maintained to prevent dust from gathering, and gloves were used whenever handling of the Mini-Flo was required. Inspite of all these efforts, small size particles, assumed to be less that $0.22 \mu \mathrm{m}$ were present. These particles can affect the fluorescence noise level by scattering extra blue light through the fiber optic. With the shortening of the fiber optic, this level disappeared.

\subsubsection{Ambient Light}

As mentioned before, when operating at maximum PMT gain, single photons can transmit noticeable voltage pulses, thereby increasing the noise level. As with cleanliness, special care was taken to keep the Mini-Flo in complete darkness. Black cloth was draped over the Mini-Flo and room lights were turned off during experimental runs. The Mini-Flo chamber walls were painted with a light absorbing black paint which greatly reduced random blue light reflections. Apertures were used to spatially filter any environmental light that could have been focused into the flow chamber as well.

\subsubsection{Electronic Noise}

Since the noise signals were in the $\mathrm{mV}$ range, the issue of environmental electronic noise had to be addressed. Before any experiments were performed, ground loops and various RF noises were investigated. 
Ground loops on the optical bench existed between the laser and PMT power sources; these were eliminated through proper insulation. The major RF noise disturbance emanated from the Cytomation data-acquisition system; this problem was resolved by placing it farther away from the Mini-Flo. 


\subsection{Discussion \&'Other Issues}

As mentioned before in section 1.0, the primary concern of this thesis was to improve the signal/noise ratio of the Mini-Flo for use in Dr. Vyas' experimentation with a bead based fluorescence assay for HIV infected blood. For the purposes of accuracy, Dr. Vyas desired an inexpensive flow cytometer whose fluorescence to non-fluorescence signal ratio was greater than or equal to the commercially available FacScan (which has a signal ratio of 100).

The systematic investigation and experimentation of all possible noise sources in the Mini-Flo improved the signal ratio from 8 to 75 . The major source of fluorescence noise was discovered to be the fiber optic. Unfortunately, this was the last noise experiment performed on the Mini-Flo; as a result, relatively small fluorescence noise improvements in the previous experiments could have been masked by the large noise levels of the long fiber optic. With the time constraints on the project, the previous experiments could not be performed again. In particular, the lengthening of the distance between the PMT and the optical surfaces might have shown better results. There is no question that all the optical surfaces fluoresce from $476.5 \mathrm{~nm}$ light; the concern is whether this fluorescence is great enough to significantly affect PMT noise levels. Had the fiber length been decreased first before performing this experiment, further improvements might have been noticed. The following section summarizes all improvements on the current 
Mini-Flo by explaining what design changes need to be made in order to guarantee signal/noise ratio improvements.

\subsection{The New Mini-Flo (A Design Discussion)}

The most significant design change required is shortening of the fiber optic. This can be done by placing the optics package with PMT directly underneath the flow chamber. Not only can the fiber length be reduced to about $1^{\prime \prime}$, but it will be straight and have no bends, thereby reducing the attenuation of signal. The flow chamber area should also be scaled down so that the fiber is millimeters away from the nozzle exit. The primary advantages of this are lowered coefficient of variation $(\mathrm{CV})$ and greater signal size. At small distances away from the nozzle exit, the particles tend to stay better aligned in the center of the sheath. Thus, a focused laser beam will consistently illuminate each bead with the same amount of light, and reduce the coefficient of variation. In addition, if the laser-particle intersection point is closer to the polished fiber optic, the light has less distance to travel through the liquid wave guide, and signal attenuation is reduced.

Another major change that needs to occur is switching the laser source from a $476.5 \mathrm{~nm}$ line to $488 \mathrm{~nm}$. Throughout the vast field of bio-fluorescence applications, the conventional excitation source for fluroescein is $488 \mathrm{~nm}$ 
light. The reason for this is because $488 \mathrm{~nm}$ is closer to fluorescein's peak absorbance than $476.5 \mathrm{~nm}$. This change in excitation does not constitute any major changes in the optical processing package; in fact, it facilitates the design. $488 \mathrm{~nm}$ line filters are more readily available and cheaper than $476.5 \mathrm{~nm}$ line filters. Thus, with a $488 \mathrm{~nm}$ laser source, $488 \mathrm{~nm}$ line filters can replace the $480 / 40$ bandpass filter (placed before the Mini-Flo) and the $500 \mathrm{~nm}$ low pass filter (placed between the dichroic and the side scatter photo-diode); all other optics will remain the same (see Figure 5.1).

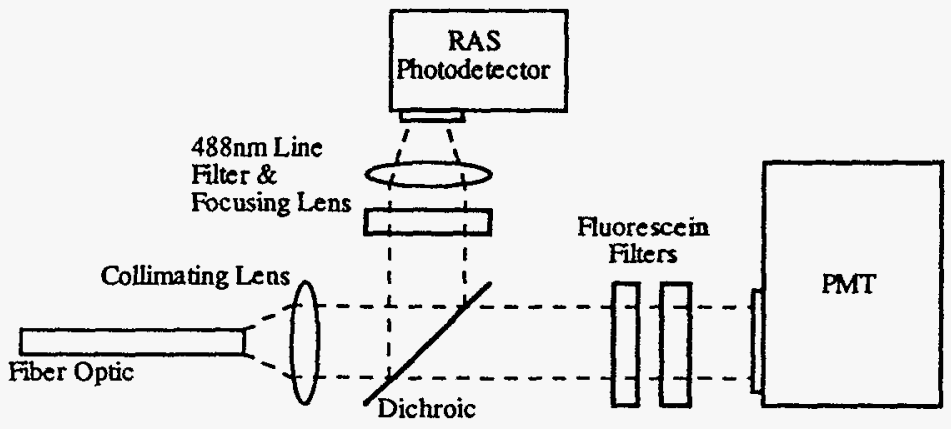

Figure 5.1 - Modified optics package schematic

The electronics data-acquisition system used for these experiments was a Stanford Research System ${ }^{\oplus}$ (SRS) current pre-amplifier feeding into a Cytomation ${ }^{\otimes}$ Cicero pulse peak-height detector. The Cicero system could measure pulses up to ten volts over 256 channels (thereby giving a resolution of $\sim 40 \mathrm{mV}$ ), whereas the SRS pre-amplifier had a saturation voltage of only about 3 volts. Therefore, only about 75 channels could be used on the Cicero 
system. The pre-amplifiers also had poor noise filtration circuitry as well as low linear gain; improvements can be made here by using low noise log amplifiers with high ( 10V) saturation voltages. Figure 5.2 shows a schematic of the new modifications to the data acquisition system. Rather than using the noise filtration electronics in the pre-amplifiers, a dedicated bandpass is placed before entering the pre-amplifier. Therefore, log gain will only be applied to scatter or fluorescence signals, and other noise frequencies will be eliminated.

Fluorescent PMT Log Pre-Amp

Signal

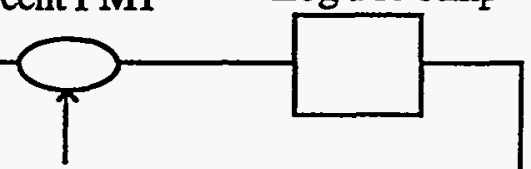

Dedicated Fluorescein \& Elastic Light signal bandpass filters

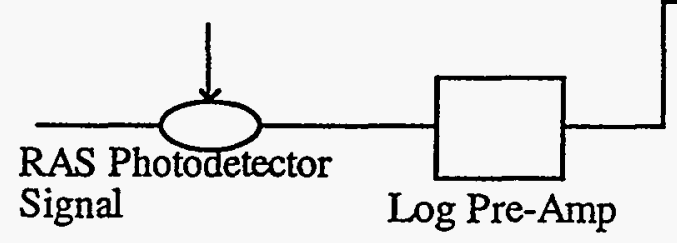
Cicero $^{\circ}$ Box

Figure 5.2 - Modified electronics schematic 


\subsection{Issues for Further Studies}

The Mini-Flo's main improvements obviously lie in the increased fluorescence signal-to-noise ratio; however, as the experimentation proceeded, new issues surfaced which need to be addressed in the future, in order to improve Mini-Flo performance.

\subsubsection{Fiber Optic End Polishing}

Ideally, if the fiber optic is conically polished, maximum signals will be detected when the center of the flow stream is roughly in line with the center of the fiber optic (see Figure 5.3). During the experimentation process however, this was not the case. In fact, signals were consistently stronger when the flow stream center was moved $\sim 25 \mu \mathrm{m}$ off center. The reason for this is improper conical polishing; since there is only a $220 \mu \mathrm{m}$ diameter of core material, conical polishing slightly off-axis can result in poor signal transmission. The maximum numerical aperture of the fiber optic is not properly utilized in this case, and as a result, signal transmission is decreased. 


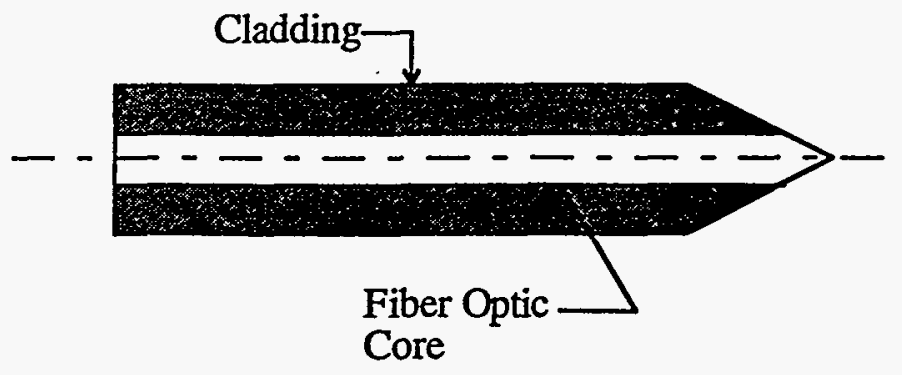

Figure 5.3 - Ideal fiber optic conical polish

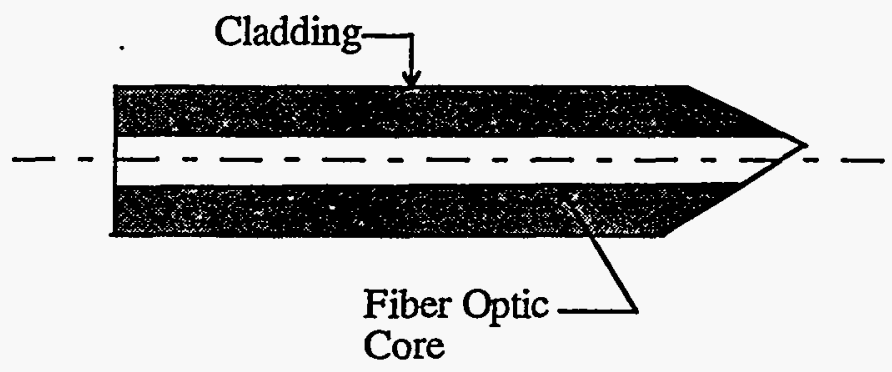

Figure 5.4 - Actual fiber optic conical polish

In order to rectify the situation, further studies must be performed on improving accuracy of fiber optic tip polishing. Presently, for the purposes of this experiment, the fiber optic was polished using a millimeter precision polishing machine. When light is sent through the fiber optic from the other end, the light traces out an improper polishing job consistent with Figure 5.4 as opposed to 5.3 . 


\subsubsection{Flow Over Conical Tip}

In addition to accurate polishing, flow over the tip is just as important. The slightest vibration or disturbance can prevent light from traveling down the liquid waveguide; therefore further studies must be performed to determine the best flow conditions for maximum signal/noise ratio. Currently, preliminary calculations have been performed in order to ensure proper laminar flow over the tip. However, the detailed dynamics of such a flow have not been extensively studied. For example, it is possible that as the flow comes out of the nozzle, the surface is not exactly cylindrical, but might have microscopic ripples resulting from the pressure and inertial changes. As a result, improper surface geometry of the liquid waveguide can bring about higher attenuation of the light signal.

The only way to understand the dynamics of this problem is to model the flow on a computer. Using Newton's equations for fluid flow and the initial conditions, the exact surface quality of the flow can be determined, and improved. 


\subsubsection{Particle Rates}

When - a particle passes through the laser intersection point, it sends some elastically and inelastically scattered light down through the liquid waveguide. At this time, if particles are present downstream of the intersection point, a portion of this scattered light will be spatially apertured out. In addition, elastically scattered light hitting downstream particles will excite inelastically scattered light as well, thereby creating false signal throughputs.

Unfortunately, this problem is highly dependent on exact locations of particles, thus making it extremely difficult to solve. In the liquid waveguide, elastic light scattered from a point source is totally internally reflected downwards. Depending on where exactly the particles downstream are, the scattered light can be either entirely or partially apertured out. The situation is complicated by the fact that the particles coming down are asynchronous; while the particle rate might be constant, the time interval, and therefore distance, between particles are quite random. Particle locations downstream of the laser intersection point are difficult to determine; thus, attempting to find a correlation between particle rate and signal throughput is extremely complicated (if at all possible). 


\subsection{Conclusion}

As stated before in the introduction, the motivation for this project was to achieve a signal/noise ratio greater than or equal to the commercially available FacScan, for clinical use with the HIV-probe bead based assay. Through a series of experiments, the signal/noise ratio was increased from 8 to 75 . The most significant improvements were made during the last series of experiments which investigated auto-fluorescence of the fiber optic. Sensitivity has improved, and auto-fluorescence of the Polyscience and FCS non-fluorescent beads are now at an almost undetectable level. In retrospect, it would have been much better to perform the fiber optic length experiments first since they had such a dramatic affect on the fluorescence noise level. However, due time restrictions, previous experiments could not be run again with the shorter fiber.

At a gain of 75, while it might be a significant improvement, the MiniFlo is still not at a level to be used with this bead-based assay (another factor of 2 improvement is still desired). In order to accomplish this, the source of the $8 \mathrm{mV}$ in Figure 4.13 must be investigated and removed. Most probably, its source will be in the 3 " of fiber optic remaining. However, by changing the fiber material to Suprasil and making it short and straight (as discussed in section 5), this will probably be eliminated. In addition, by correcting the other two major weaknesses in this system (i.e. $488 \mathrm{~nm}$ light instead of $476.5 \mathrm{~nm}$ and 
clean log pre-amplifiers instead of noisy linear ones), the Mini-Flo could well surpass the FacScan's performance. 


\section{Bibliography}

1. Hodkinson, J. and I. Greenleaves Computations of Light-Scattering and Extinction by Spheres According to Diffraction and Geometrical Optics, and Some Comparisons with Mie Theory Journal of the Optical Society of America, Vol. 53, No. 5 (1963).

2. Ippen, E. and R. Stolen Stimulated Brillouin Scattering in Optical Fibers, Appl. Phys. Lett., Vol. 21, No. 11 (1972).

3. Kerker, M. Elastic and Inelastic Light Scattering in Flow Cytometry Cytometry 4:1-10 (1983).

4. Laerum, O., D. et al (editors) Flow Cytometry IV Columbia University Press, New York (1980).

4. Mariella Jr., Raymond, P. et al Aqueous Carrier Waveguide in a Flow Cytometer, Lawrence Livermore National Laboratory, University of California, Livermore, California, (1995, U.S. Patent 5,475,487).

6. Mayall, Brian Cytometry in the Clinical Laboratory: Quo Vadis? Annals New York Academy of Sciences (1969).

7. Melamed, Myron, R. et al (editors) Flow Cytometry and Sorting, John Wiley \& Sons, New York (1979).

8. Mullaney, P. at al Cell Sizing: A Light Scattering Photometer for Bapid Volume Determination, Rev. Scie. Instr., Vol. 40, No. 8 (1969). 
9. Mullaney, P. and P. Dean The Small Angle of Light Scattering of Bilogical Cells: Theoretical Considerations, Biophysical Journal, Vol. 10, No. 8 (1970).

10. Ormerod, M., G. (editors) Flow Cytometry: A Practical Approach, IRL Press, Oxford (1990).

11. Radbruch, A. (editors) Flow Cytometry and Cell Sorting, Springer-Verlag, New York (1992).

12. Shapiro, H. and M. Hercher Flow Cytometers Using Optical Waveguides in Place of Lenses for Specimen Illumination and Light Collection, Cytometry 7:221-223 (1986).

13. Shapiro, H. Practical Flow Cytometry (3rd Edition), Wiley-Liss, New York (1995).

14. Shapiro, H. Trends and Developments in Flow Cytometry Instrumentation, Annals New York Academy of Sciences (1970).

15. Stolen, H., E. Ippen, and A. Tynes Raman Oscillations in Glass Optical Waveguide, Appl. Phys. Lett., Vol. 20, No. 2 (1972).

16. Taylor, Lansing, D. et al (editors) Applications of Fluorescence in the Biomedical Sciences, Alan R. Liss, Inc., New York (1986).

17. Vyas, G. N. et al Early Detection of Antibodies Against rDNA-Produced HIV Proteins with a Flow Cytometric Assay, Blood, Vol. 73, No. 7 (1989).

18. Vyas, G. N. et al Flow Cytometric Immunodetection of Human Immunodeficiency Virus Type 1 Proviral DNA by Heminested PCR and Digoxigenin-Labeled Probes, Clin. Diagn. Lab. Immunol., Vol. 1, No. 1 (1994). 
19. Watson, James, V. Flow Cytometry Data Analysis: Basic Concepts and Statistics, Cambridge University Press, Oakleigh, Victoria (1992). 